\title{
Real frontiers of fake planes
}

\author{
Adrien Dubouloz $^{1}$. Frédéric Mangolte ${ }^{2}$
}

Received: 8 September 2015 / Accepted: 11 November 2015 / Published online: 25 November 2015

(C) Springer International Publishing AG 2015

\begin{abstract}
In Dubouloz and Mangolte (Fake real planes: exotic affine algebraic models of $\mathbb{R}^{2}$, arXiv:1507.01574, 2015), we define and partially classify fake real planes, that is, minimal complex surfaces with conjugation whose real locus is diffeomorphic to the euclidean real plane $\mathbb{R}^{2}$. Classification results are given up to biregular isomorphisms and up to birational diffeomorphisms. In this note, we describe in an elementary way numerous examples of fake real planes and exhibit examples of such planes of every Kodaira dimension $\kappa \in\{-\infty, 0,1,2\}$ which are birationally diffeomorphic to $\mathbb{R}^{2}$.
\end{abstract}

Keywords Real algebraic model $\cdot$ Affine surface $\cdot$ Rational fibration - Birational diffeomorphism - Affine complexification

Mathematics Subject Classification $14 \mathrm{R} 05 \cdot 14 \mathrm{R} 25 \cdot 14 \mathrm{E} 05 \cdot 14 \mathrm{P} 25 \cdot 14 \mathrm{~J} 26$

\section{Introduction}

A complexification of a real smooth $\mathrm{C}^{\infty}$-manifold $M$ is a real algebraic manifold $S$ (see terminological conventions at the beginning of Sect. 2) whose real locus is

This project was partially funded by ANR Grant "BirPol” ANR-11-JS01-004-01.

Frédéric Mangolte

frederic.mangolte@univ-angers.fr

Adrien Dubouloz

adrien.dubouloz@u-bourgogne.fr

1 Institut de Mathématiques de Bourgogne, UMR 5584, CNRS, Université Bourgogne

Franche-Comté, 21000 Dijon, France

2 LUNAM Université, LAREMA, Université d'Angers, 49045 Angers, France 
diffeomorphic to $M: S(\mathbb{R}) \approx M$. Some manifolds such as real projective spaces $\mathbb{R P}^{n}$ and real euclidean affine spaces $\mathbb{R}^{n}$ have natural algebraic complexifications, given by the complex projective and affine spaces $\mathbb{C} \mathbb{P}^{n}$ and $\mathbb{C}^{n}$ respectively. But these also admit infinitely many other complexifications, and it is a natural problem to try to classify them up to appropriate notions of equivalence. We focus on the case when $M=\mathbb{R}^{2}$ which is already surprisingly involved. Consider the following two examples:

- Start from $S_{0}=\mathbb{C}_{u, v}^{2}$ with the usual conjugation $(u, v) \mapsto(\bar{u}, \bar{v})$. We have $S_{0}(\mathbb{R})=\mathbb{R}^{2}$. Blowing-up a pair of conjugated nonreal points of $\mathbb{C}^{2}$, we get a surface $S_{1}$ which is not isomorphic to $\mathbb{C}^{2}$, actually not even affine as it contains proper curves, but $S_{1}(\mathbb{R}) \approx \mathbb{R}^{2}$.

- Start from $\mathbb{C P}_{x: y: z}^{2}$ and choose a pair of conjugated lines $L, \bar{L}$ meeting at a point on the line at infinity $L_{\infty}=\{z=0\}$. Let $S_{2}$ be the complement in $\mathbb{C P}^{n}$ of the union $L \cup \bar{L} \cup L_{\infty}$. Then $S_{2}$ is isomorphic to $\mathbb{C} \backslash\{$ a pair of conjugated points $\} \times \mathbb{C}$ and $S_{2}(\mathbb{R}) \approx \mathbb{R}^{2}$.

In the two cases above, the complexification is topologically far from $\mathbb{C}^{2}$. So we will seek for topologically minimal complexifications of $\mathbb{R}^{2}$ which we call fake real planes.

Definition 1.1 A nonsingular quasi-projective complex algebraic surface $S$ endowed with an anti-holomorphic involution is called a fake real plane if:

- $S$ is a real plane: $S(\mathbb{R}) \approx \mathbb{R}^{2}$;

- $S$ is topologically minimal: $H_{k}(S ; \mathbb{Q})=0$ for all $k \geqslant 1$;

- $S$ is fake: $S$ is not biregularly isomorphic to $\mathbb{C}^{2}$ as a real algebraic surface.

Every fake real plane $S$ is affine and rational over $\mathbb{R}$, see Sect. 2.1. In the projective setting, we obtain complexifications of $\mathbb{R P}^{2}$ in the same way as described in the first example above by blowing-up pairs of conjugated nonreal points of $\mathbb{C P}^{2}$. Among the rational projective complexifications of $\mathbb{R P}^{2}$, the only minimal one is $\mathbb{C P}^{2}$ because it is the only one with Picard number $\rho=1$. In fact, $\mathbb{C P}^{2}$ is the only topologically minimal complexification of $\mathbb{R P}^{2}$ : there exist other smooth complex surfaces with the same homology as $\mathbb{C P}^{2}$, but none of them admits a real structure [15]. In the light of these observations, a first natural question is:

Question 1.2 Is there any fake real plane at all?

An affirmative answer to Question 1.2 is given in the article [8], where we introduce and partially classify fake real planes. In the present paper, as an application of general classification results in [8], we describe in an explicit way numerous examples.

Let $S$ and $S^{\prime}$ be two complexifications of a given topological surface $M$. We say that $S$ and $S^{\prime}$ are $\mathbb{R}$-biregularly birationally equivalent if their real loci $S(\mathbb{R})$ and $S^{\prime}(\mathbb{R})$ have isomorphic Zariski open neighborhoods in $S$ and $S^{\prime}$, respectively. Equivalently the surfaces $S(\mathbb{R})$ and $S^{\prime}(\mathbb{R})$ are birationally diffeomorphic, that is:

Definition 1.3 Let $S$ and $S^{\prime}$ be real algebraic surfaces. Their real loci $S(\mathbb{R})$ and $S^{\prime}(\mathbb{R})$ are birationally diffeomorphic if there is a diffeomorphism $f: S(\mathbb{R}) \rightarrow S^{\prime}(\mathbb{R})$ which extends as a real birational map $\psi: S \rightarrow S^{\prime}$ whose indeterminacy locus does not intersect $S(\mathbb{R})$, and such that the indeterminacy locus of $\psi^{-1}$ does not intersect $S^{\prime}(\mathbb{R})$. 
A real algebraic surface $S$ is rectifiable if its real locus $S(\mathbb{R})$ is birationally diffeomorphic to $\mathbb{R}^{2}$.

It is well known that every rational projective complexification of $\mathbb{R} \mathbb{P}^{2}$ is $\mathbb{R}$-biregularly birationally equivalent to $\mathbb{R P}^{2}$, even dropping the topological minimality condition, see [16]. Thus next natural questions are:

Question 1.4 Is there any rectifiable fake real plane?

Question 1.5 Let $S$ be a fake real plane. Is $S$ rectifiable?

Again the answer to Question 1.4 is affirmative; examples of rectifiable fake real planes of Kodaira dimensions $-\infty$ and 0 were given in [8]. In the present paper, we exhibit infinite families of rectifiable fake real planes in every Kodaira dimension. In spite of this, Question 1.5 remains open.

The idea of this paper emerges thanks to the conference Frontiers of Rationality which took place in Spitsbergen (Norway) in July 2014. The word "frontier" in our title is an attempt to give credit to this exceptional event.

\section{Generalities on fake real planes}

\section{Terminological conventions}

- A real algebraic manifold of dimension $n$ is a quasi-projective complex algebraic manifold $S$ of complex dimension $n$ endowed with an anti-holomophic involution whose set of fixed points is called the real locus and denoted by $S(\mathbb{R})$. A real map is a complex map commuting with involutions. A real algebraic surface is a real algebraic manifold of dimension 2 . The manifolds $S$ and $S(\mathbb{R})$ are assumed to be endowed with their euclidean topology.

- A topological surface is a real 2-dimensional $\mathrm{e}^{\infty}$-manifold. By our convention, a real algebraic surface $S$ is nonsingular; as a consequence, if nonempty, the real locus $S(\mathbb{R})$ gets a natural structure of a topological surface when endowed with the euclidean topology. Furthermore $S(\mathbb{R})$ is compact if $S$ is projective.

\subsection{Generalities on topologically minimal real planes}

By virtue of results of Fujita [9] and Gurjar-Pradeep-Shastri [12,13] every smooth $\mathbb{Q}$-acyclic complex surface $S$ is affine and rational. If $S$ is real, then it admits a real completion $S \hookrightarrow(V, B)$ into a smooth real projective surface $V$ with connected real boundary curve $B=V \backslash S$. In the case where $S$ is a real plane, the connectedness of $S(\mathbb{R}) \approx \mathbb{R}^{2}$ implies that $V$ is a rational complex surface with connected real locus, hence an $\mathbb{R}$-rational real projective surface, i.e. a real surface admitting a real birational map $V \rightarrow \mathbb{C P}^{2}$ to $\mathbb{C P}^{2}$ equipped with its standard real structure. The free abelian group $\mathbb{Z}\langle B\rangle$ generated by the irreducible components of $B$ and the divisor class group $\mathrm{Cl}(V)$ of $V$ both inherit a structure of $G$-module for the group $G=\{1, \sigma\} \simeq \mathbb{Z}_{2}$ generated by the real structure $\sigma$ on $V$. The inclusion $j: B \hookrightarrow V$ gives rise to a homomorphism $j_{*}: \mathbb{Z}\langle B\rangle \rightarrow \mathrm{Cl}(V)$ of $G$-module, hence to an induced homomorphism 
$H^{2}\left(j_{*}\right): H^{2}(G, \mathbb{Z}\langle B\rangle) \rightarrow H^{2}(G, \mathrm{Cl}(V))$ of $\mathbb{Z}_{2}$-vector spaces between the Galois cohomology groups $H^{2}(G, M)=\operatorname{Ker}\left(\operatorname{id}_{M}-\sigma\right) / \operatorname{Im}\left(\operatorname{id}_{M}+\sigma\right), M=\mathbb{Z}\langle B\rangle, \operatorname{Cl}(V)$. The next theorem will be the most useful for the constructions presented below.

Theorem 2.1 Let $(V, B)$ be a pair consisting of an $\mathbb{R}$-rational projective real surface $V$ and a real curve $B \subset V$. Then the surface $S=V \backslash B$ is an $A$-acyclic, where $A=\mathbb{Z}$ or $\mathbb{Q}$, real plane if and only if the conditions are satisfied:

(a) $V$ and $B$ are connected and simply connected and $j_{*} \otimes_{\mathbb{Z}} A: \mathbb{Z}\langle B\rangle \otimes_{\mathbb{Z}} A \rightarrow \mathrm{Cl}(V)$ $\otimes_{\mathbb{Z}} A$ is an isomorphism.

(b) The real locus of $B$ is nonempty and $H^{2}\left(j_{*}\right): H^{2}(G, \mathbb{Z}\langle B\rangle) \rightarrow H^{2}(G, \mathrm{Cl}(V))$ is an isomorphism.

Proof The first assertion is essentially a rephrasing of a classical criterion established first by Ramanujam [20] and Fujita [9] which asserts that the complex surface $S$ is $A$-acyclic if and only if $V$ and $B$ are connected and the homomorphism $H_{2}(B ; A) \rightarrow$ $H_{2}(V ; A)$ induced by the inclusion $B \hookrightarrow V$ is an isomorphism. Indeed, $H_{2}(B ; A)$ is a free abelian group isomorphic to $\mathbb{Z}\langle B\rangle \otimes_{\mathbb{Z}} A$ via the map which associates to every irreducible component of $B$ its fundamental class in homology while the isomorphism $H_{2}(V ; A) \simeq \mathrm{Cl}(V) \otimes_{\mathbb{Z}} A$ follows from that fact that since $V$ is rational, the cycle map $\mathrm{Cl}(V) \rightarrow H_{2}(V, \mathbb{Z})$ which associates to every irreducible complex curve $D \subset V$ its fundamental class is an isomorphism. The second assertion essentially follows from the combination of the well-known fact that a relatively compact topological surface $M \subset \bar{M}$ with connected boundary $\bar{M} \backslash M$ is diffeomorphic to $\mathbb{R}^{2}$ if and only if it is connected and $\mathbb{Z}_{2}$-acyclic with the cycle map construction due to Borel-Haefliger [3], see $[8$, Section 2].

Remark 2.2 (i) The curve $B$ in the previous proposition need not be an SNC divisor on $V$, but the criterion implies in particular that if $S=V \backslash B$ is $A$-cyclic then its inverse image $\tau^{-1}(B)$ in a log-resolution $\tau: V^{\prime} \rightarrow V$ of the pair $(V, B)$ defined over $\mathbb{R}$ is a real tree of rational curves, that is a tree of rational curves with an induced action of the real structure $\sigma$ on $V^{\prime}$. It follows in particular that the real locus of $B$ is either empty or a connected union of curves homeomorphic to a circle.

(ii) In the case where $j_{*} \otimes_{\mathbb{Z}} \mathbb{Q}: \mathbb{Z}\langle B\rangle \otimes_{\mathbb{Z}} \mathbb{Q} \rightarrow \mathrm{Cl}(V) \otimes_{\mathbb{Z}} \mathbb{Q}$ is an isomorphism, one infers from the long exact sequence of relative homology for the pair $(V, B)$ that $H_{1}(S, \mathbb{Z})$ is a torsion group isomorphic to $\mathrm{Cl}(V) / \operatorname{Im}\left(j_{*}\right)$.

Example 2.3 The complement $S$ of a smooth real conic $B$ in $\mathbb{C P}^{2}$ is a $\mathbb{Q}$-acyclic real surface which is a not a real plane. It can be seen directly that $S(\mathbb{R})$ is either diffeomorphic to $\mathbb{R} \mathbb{P}^{2}$ if $B(\mathbb{R})=\varnothing$ or to the disjoint union of $\mathbb{R}^{2}$ with a Möbius band otherwise. In the setting of Theorem 2.1, the image of the generator $[B]$ of $\mathbb{Z}\langle B\rangle \simeq \mathbb{Z} \cdot[B] \simeq H_{2}(B ; \mathbb{Z})$ by $j_{*}: \mathbb{Z}\langle B\rangle \rightarrow \mathrm{Cl}\left(\mathbb{C P}^{2}\right) \simeq H_{2}\left(\mathbb{C P}^{2} ; \mathbb{Z}\right) \simeq \mathbb{Z} \cdot[\ell]$, where $[\ell]$ denotes the class of a real line, is equal to $2 \cdot[\ell]$. So $S$ is $\mathbb{Q}$-acyclic,

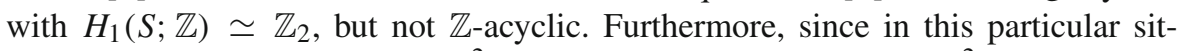
uation we have isomorphisms $H^{2}(G, \mathbb{Z}\langle B\rangle) \simeq \mathbb{Z}\langle B\rangle \otimes_{\mathbb{Z}} \mathbb{Z}_{2}$ and $H^{2}(G, \mathrm{Cl}(V)) \simeq$ $\mathrm{Cl}(V) \otimes_{\mathbb{Z}} \mathbb{Z}_{2}, H^{2}\left(j_{*}\right): H^{2}(G, \mathbb{Z}\langle B\rangle) \rightarrow H^{2}(G, \mathrm{Cl}(V))$ is then the trivial map. 


\section{Fake planes of negative Kodaira dimension}

It turns out that $\mathbb{C}^{2}$ equipped with its standard real structure is the only $\mathbb{Z}$-acyclic real plane $S$ of negative Kodaira dimension. Indeed, a complex $\mathbb{Z}$-acyclic surface of negative Kodaira dimension is isomorphic to $\mathbb{C}^{2}$, by virtue of [18], while every real structure on $\mathbb{C}^{2}$ is isomorphic to the standard one, as a consequence of [14]. In this section, we briefly review general geometric properties of $\mathbb{Q}$-acyclic fake planes. Then, as a particular instance of a large class of such planes which are known since [8] to be $\mathbb{R}$-biregularly birationally equivalent to $\mathbb{C}^{2}$, see Theorem 3.3 , we construct a pair of nonisomorphic $\mathbb{Q}$-acyclic fake planes of negative Kodaira dimension with the same homology groups, whose real loci are both birationally diffeomorphic to $\mathbb{R}^{2}$.

\subsection{Basic properties of $\mathbb{Q}$-acyclic fake planes of negative Kodaira dimension}

By virtue of [18], the negativity of the Kodaira dimension of a smooth complex affine surface $S$ is equivalent to the existence of an $\mathbb{A}^{1}$-fibration $\rho: S \rightarrow C$, that is, a fibration with general fibers isomorphic to $\mathbb{C}$, over a smooth curve $C$. One direction is clear for if $\rho: S \rightarrow C$ is such a fibration, then $C$ contains a nonempty Zariski open subset $C_{0}$ over which $\rho$ restricts to a trivial bundle $C_{0} \times \mathbb{C}$, and so $\kappa(S) \leqslant \kappa\left(C_{0} \times \mathbb{C}\right)=-\infty$ by Iitaka's easy addition formula. When $S$ is in addition real, there is no reason in general that the existing $\mathbb{A}^{1}$-fibration $\rho: S \rightarrow C$ is a real map: for instance, the complement of a smooth conic $B$ in $\mathbb{C P}^{2}$ with empty real locus is a real surface of negative Kodaira dimension without any real $\mathbb{A}^{1}$-fibration $\rho: S \rightarrow C$. Indeed, if such a fibration existed then the closure in $\mathbb{C P}^{2}$ of its fiber over a general real point of $C$ would be a real rational curve intersecting $B$ in a unique point, necessarily real, which is impossible. The following theorem shows in particular that this phenomenon does not occur for real planes.

Theorem 3.1 ([8, Theorem 4.1]) For a smooth affine real surface $S$ the following are equivalent:

- $S$ is a $\mathbb{Q}$-acyclic real plane of negative Kodaira dimension.

- $S$ admits a real $\mathbb{A}^{1}$-fibration $\rho: S \rightarrow \mathbb{C}$ whose closed fibers are all isomorphic to $\mathbb{C}$ when equipped with their reduced structure and whose fibers over the real locus of $\mathbb{C}$ have odd multiplicities.

Example 3.2 Let $s \geqslant 1$, let $[m]=\left(m_{1}, \ldots, m_{s}\right)$ be a collection of integers $m_{i} \geqslant 2$, let $[p]=\left(p_{1}, \ldots, p_{s}\right)$ be a collection of odd integers $p_{i} \geqslant 3$ and let $r_{1}, \ldots, r_{s} \in \mathbb{R}$ be a collection of pairwise distinct real numbers. Then the real surface $S_{s,[m][p]} \subset \mathbb{C}^{3}$ defined by the equation

$$
\prod_{i=1}^{s}\left(x-r_{i}\right)^{m_{i}} z=\sum_{i=1}^{s} \prod_{j \neq i}\left(x-r_{j}\right) y^{p_{i}}+\prod_{i=1}^{s}\left(x-r_{i}\right)
$$

is a smooth $\mathbb{Q}$-acyclic fake plane of negative Kodaira dimension. Indeed, the smoothness of $S_{s,[m][p]}$ follows for instance from the Jacobian criterion. The restriction to 
$S_{s,[m][p]}$ of the projection $\operatorname{pr}_{x}$ is a real $\mathbb{A}^{1}$-fibration $\pi: S_{s,[m][p]} \rightarrow \mathbb{C}$ with irreducible fibers, restricting to a trivial bundle over $\mathbb{C} \backslash\left\{r_{1}, \ldots, r_{s}\right\}$ and whose fiber over each of the points $r_{i}, i=1, \ldots, s$, has odd multiplicity $p_{i}$. So $S_{s,[m][p]}$ is a $\mathbb{Q}$-acyclic real plane of negative Kodaira dimension by the previous theorem.

The following partial result concerning the classification of $\mathbb{Q}$-acyclic real planes of negative Kodaira dimension up to $\mathbb{R}$-biregular equivalence was obtained in [8].

Theorem 3.3 Every $\mathbb{Q}$-acyclic real plane $S$ of negative Kodaira dimension admitting a real $\mathbb{A}^{1}$-fibration $\rho: S \rightarrow \mathbb{C}$ with at most one degenerate fiber is $\mathbb{R}$-biregularly birationally equivalent to $\mathbb{C}^{2}$.

As a consequence, the real locus of every surface $S_{1, m_{1}, p_{1}}$ as in Example 3.2 is birationally diffeomorphic to $\mathbb{R}^{2}$. We do not know whether $\mathbb{Q}$-acyclic real plane of negative Kodaira dimension $S$ admitting real $\mathbb{A}^{1}$-fibrations $\rho: S \rightarrow \mathbb{C}$ with more than one degenerate fiber is $\mathbb{R}$-biregularly birationally equivalent to $\mathbb{C}^{2}$, in particular:

Question 3.4 Is the real locus of a surface $S_{s,[m][p]}$ with $s \geqslant 2$ as in Example 3.2 birationally diffeomorphic to $\mathbb{R}^{2}$ ?

\subsection{Two examples of $\mathbb{Q}$-acyclic fake planes with real loci birationally diffeomorphic to $\mathbb{R}^{2}$}

\subsubsection{Construction and first properties}

I. First we let $S$ be the complement of a real cuspidal cubic $B$ in $V=\mathbb{C P}^{2}$. Since $B$ is equivalent in $\mathrm{Cl}(V)$ to three times the class of a real line $\ell \subset \mathbb{C P}^{2}$, the $\mathbb{Q}$ acyclicity of $S$ immediately follows from Theorem 2.1 (a) applied to the pair $(V, B)$, and we have $H_{1}(S ; \mathbb{Z}) \simeq \mathbb{Z}_{3}$ by Remark 2.2 (ii). The fact that $S$ is a real plane follows from Theorem 2.1 (b) after noting that similarly as in Example 2.3, $H^{2}(G, \mathbb{Z}\langle B\rangle) \simeq$ $\mathbb{Z}\langle B\rangle \otimes_{\mathbb{Z}} \mathbb{Z}_{2} \simeq \mathbb{Z}_{2} \cdot[B]$ and $H^{2}\left(G, \mathrm{Cl}\left(\mathbb{C P}^{2}\right)\right) \simeq \mathrm{Cl}\left(\mathbb{C P}^{2}\right) \otimes_{\mathbb{Z}} \mathbb{Z}_{2} \simeq \mathbb{Z}_{2} \cdot[\ell]$. Alternatively, one can observe that $B(\mathbb{R})$ is homeomorphic to a simple closed curve in $\mathbb{R P}^{2}$ whose homotopy class is a generator of $\pi_{1}\left(\mathbb{R P}^{2}\right)$, so $\mathbb{R P}^{2} \backslash B(\mathbb{R})$ is homeomorphic to an open disc, hence is diffeomorphic to $\mathbb{R}^{2}$. The restriction to $S$ of the rational pencil $V \rightarrow \mathbb{C P}^{1}$ generated by $B$ and three times its tangent $T$ at its unique singular point restricts to a real $\mathbb{A}^{1}$-fibration $\rho: S \rightarrow \mathbb{C}$ with a unique degenerate fiber of multiplicity 3 consisting of the intersection of $T$ with $S$. The real minimal resolution of the pencil $V \rightarrow \mathbb{C P}^{1}$ dominates the real minimal resolution $\tau: W \rightarrow V$ of the pair $(V, B)$, and is obtained from it by blowing-up three times the intersection point of the proper transform of $B$ with the successive total transforms of the exceptional locus of $\tau$. We denote by $\beta: \widetilde{V} \rightarrow V$ the so constructed surface and we denote by $C$ the last exceptional divisor produced by this sequence of blow-ups. The dual graph of the total transform of $B \cup T$ in $\widetilde{V}$ is depicted in Fig. 1.

II. Next we let $S^{\prime}$ be the smooth real affine cubic surface in $\mathbb{C}^{3}$ defined by the equation $x^{2} z=y^{3}-x$. Taking projective closure in $\mathbb{C P}^{3}$ with homogeneous coordinates $[x: y: z: t]$, we view $S^{\prime}$ as the complement in the normal real cubic surface $V^{\prime}=$ 


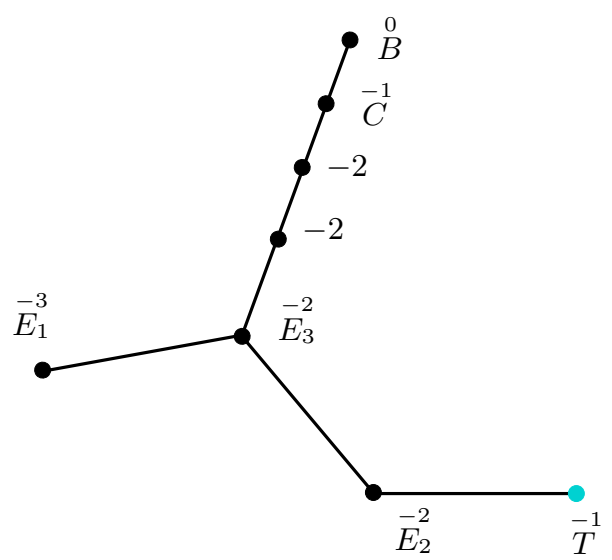

Fig. 1 Dual graph of the proper transform of $B \cup T$ in $\widetilde{V}$

$\left\{x^{2} z-y^{3}+x t^{2}=0\right\}$ of the real rational cuspidal hyperplane section $B^{\prime}=V^{\prime} \cap\{t=0\}$. Note that $V^{\prime}$ has a unique singular point of type $D_{4}$ at $[0: 0: 1: 0]$. Since the map $\mathbb{R}^{2} \rightarrow S^{\prime},(x, z) \mapsto\left(x, \sqrt[3]{x^{2} z+x}, z\right)$ is an homeomorphism between $\mathbb{R}^{2}$ and the real locus of $S^{\prime}$, it follows that $S^{\prime}$ is a real plane. The divisor class group $\mathrm{Cl}\left(V^{\prime}\right)$ of $V^{\prime}$ is isomorphic to $\mathbb{Z}$ generated by the closure $T^{\prime}$ in $V^{\prime}$ of the real line $\{x=y=0\} \subset S^{\prime}$. The divisor class group $\mathrm{Cl}\left(W^{\prime}\right)$ of the real minimal $\log$-resolution $\tau^{\prime}: W^{\prime} \rightarrow V^{\prime}$ of the pair $\left(V^{\prime}, B^{\prime}\right)$ is isomorphic to $\mathbb{Z}^{6}$ generated by the five exceptional divisors of $\tau^{\prime}$ and the proper transform of $T^{\prime}$, and since $B^{\prime} \sim 3 T^{\prime}$ in $\mathrm{Cl}\left(V^{\prime}\right)$, it follows that for an appropriate choice of bases, the map $j_{*}: \mathbb{Z}\left\langle\tau^{-1}\left(B^{\prime}\right)\right\rangle \rightarrow \mathrm{Cl}\left(W^{\prime}\right)$ is represented by a matrix $M$ of the form

$$
M=\left(\begin{array}{cc}
\operatorname{id}_{5} * \\
0 & 3
\end{array}\right) .
$$

So by virtue of Theorem 2.1 (a) applied to the real projective completion $\left(W^{\prime}, \tau^{-1}\left(B^{\prime}\right)\right)$ of $S^{\prime}, S^{\prime}$ is $\mathbb{Q}$-acyclic, with $H_{1}\left(S^{\prime} ; \mathbb{Z}\right) \simeq \mathbb{Z}_{3}$ by Remark 2.2 (ii). The restriction to $S^{\prime}$ of the projection $\operatorname{pr}_{x}$ is a real $\mathbb{A}^{1}$-fibration $\rho^{\prime}: S^{\prime} \rightarrow \mathbb{C}$ having the line $\{x=y=0\}$ as a unique degenerate fiber of multiplicity 3. So $\kappa\left(S^{\prime}\right)=-\infty$. The real minimal resolution of the pencil $V^{\prime} \rightarrow \mathbb{C P}^{1}$ induced by $\rho^{\prime}: S^{\prime} \rightarrow \mathbb{C}$ dominates the real minimal resolution $\tau^{\prime}: W^{\prime} \rightarrow V^{\prime}$ of the pair $\left(V^{\prime}, B^{\prime}\right)$, and is obtained from it by blowing-up the intersection point of the proper transform of $B^{\prime}$ with the exceptional locus of $\tau^{\prime}$. We denote by $\beta^{\prime}: \widetilde{V}^{\prime} \rightarrow V$ the so constructed surface and we denote by $C^{\prime}$ the last exceptional divisor produced by this sequence of blow-ups. The dual graph of the total transform of $B^{\prime} \cup T^{\prime}$ in $\widetilde{V}^{\prime}$ is depicted in Fig. 2.

Proposition 3.5 The surfaces $S$ and $S^{\prime}$ are nonisomorphic fake planes of negative Kodaira dimension.

Proof That $S$ and $S^{\prime}$ are fake planes of negative Kodaira dimension follows from the construction above. The fact they are nonisomorphic as complex surfaces can be deduced as follows. First since the dual graphs of the minimal resolutions of the pairs 


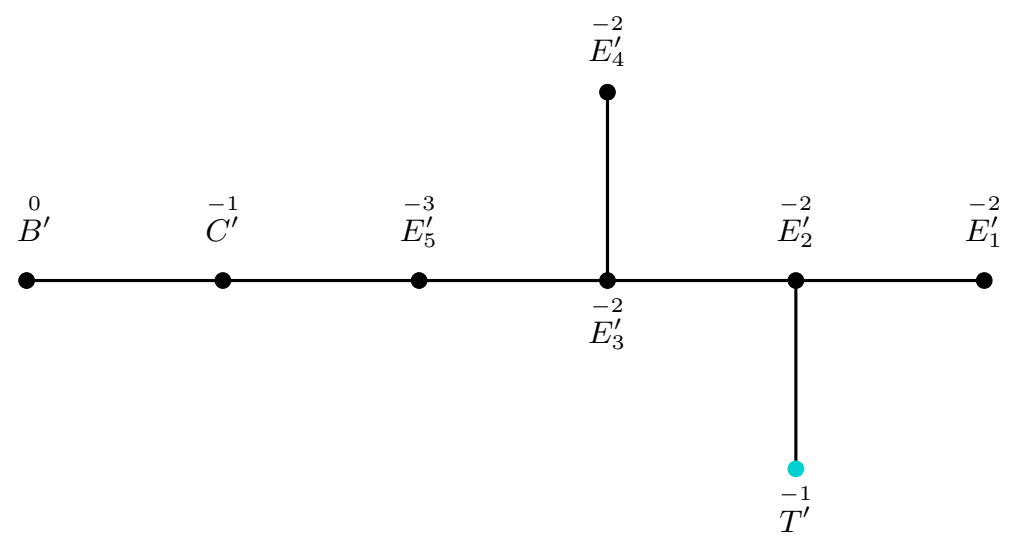

Fig. 2 Dual graph of the proper transform of $B^{\prime} \cup T^{\prime}$ in $\widetilde{V}$

$(V, B)$ and $\left(V^{\prime}, B^{\prime}\right)$ are not chains, it follows from [1] that the $\mathbb{A}^{1}$-fibrations $\rho: S \rightarrow \mathbb{C}$ and $\rho^{\prime}: S^{\prime} \rightarrow \mathbb{C}$ constructed above are unique, up to composition by automorphisms of the base $\mathbb{C}$. This implies in particular that any isomorphism $\Phi$ between $S$ and $S^{\prime}$ is an isomorphism of fibered surfaces, that is, there exists an isomorphism $\phi: \mathbb{C} \stackrel{\sim}{\rightarrow} \mathbb{C}$ such that $\rho^{\prime} \circ \Phi=\phi \circ \rho$. Every such isomorphism then admits a unique extension to a birational map $\bar{\Phi}: \widetilde{V} \rightarrow \widetilde{V}^{\prime}$ with a unique proper base point, supported at the intersection of $C$ with the proper transform of $B$, restricting to an isomorphism between the complements $\widetilde{V} \backslash \beta_{*}^{-1}(B)$ and $\widetilde{V}^{\prime} \backslash\left(\beta^{\prime}\right)_{*}^{-1}\left(B^{\prime}\right)$ of the proper transforms of $B$ and $B^{\prime}$ respectively and mapping $C$ isomorphically onto $C^{\prime}$ (see e.g. [2] where these birational maps are called fibered modifications). But the fact that the irreducible components of $\beta^{-1}(B) \backslash \beta_{*}^{-1}(B)$ and $\beta^{-1}\left(B^{\prime}\right) \backslash \beta_{*}^{-1}\left(B^{\prime}\right)$ intersecting $C$ and $C^{\prime}$ respectively have different self-intersections prevents the existence of any such birational map. So $S$ and $S^{\prime}$ are not isomorphic.

Remark 3.6 The surfaces $S$ and $S^{\prime}$ do not only have the same homology group but also have the same fundamental group $\pi_{1} \simeq \mathbb{Z}_{3}$. In fact, it can be shown that the complex algebraic threefolds $S \times \mathbb{A}^{1}$ and $S^{\prime} \times \mathbb{A}^{1}$ are isomorphic, so that $S$ and $S^{\prime}$ are in particular homotopically equivalent. A direct computation reveals further that the fundamental groups at infinity of $S$ and $S^{\prime}$ (see e.g. [17, Section 4.9, p. 246] for the definition and an algorithm for the computation of these groups) are both isomorphic to $\mathbb{Z}_{9}$. We do not know whether $S$ and $S^{\prime}$ are homeomorphic as real 4-manifolds or not.

\subsubsection{Birational diffeomorphisms}

Here we show that the real loci of $S$ and $S^{\prime}$ are not only diffeomorphic to $\mathbb{R}^{2}$ but actually birationally diffeomorphic to it.

I. In the minimal real log-resolution $\tau: W \rightarrow V$ of the pair $(V, B)$, the proper transform of any pair $(\ell, \bar{\ell})$ of general nonreal complex conjugate lines in $V=\mathbb{C P}^{2}$ passing through the singular point $p_{0}$ of $B$ consists of a pair of complex conjugate rational 0 - 

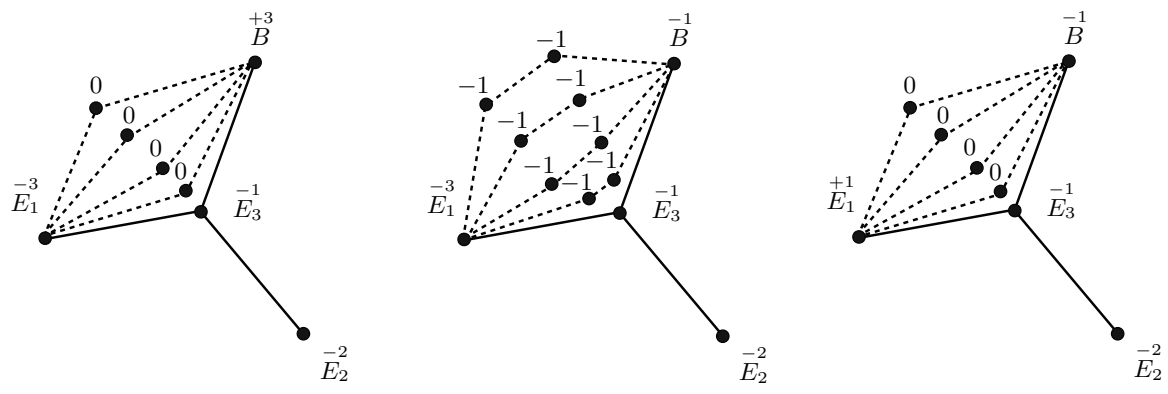

Fig. 3 Elementary transformations

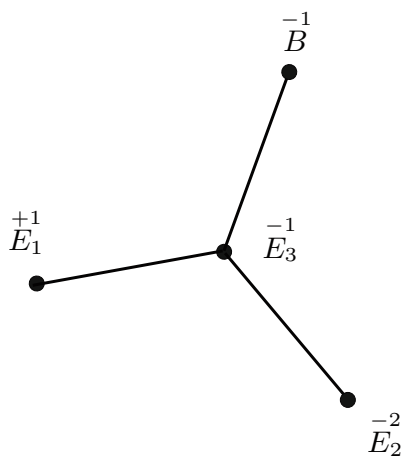

Fig. 4 Dual graph of the proper transform of $\tau^{-1}(B)$ by $\theta_{1}$

curves intersecting the proper transform of $B$ transversally in a pair of nonreal complex conjugate points $(q, \bar{q})$. Choosing two distinct such general pairs $\left(\ell_{i}, \bar{\ell}_{i}\right), i=1,2$, we let $\theta_{1}: W \rightarrow W_{1}$ be the real birational map consisting of the blow-up of the corresponding two pairs of points $\left(q_{i}, \bar{q}_{i}\right), i=1,2$, followed by the contraction of the proper transforms of $\ell_{i}$ and $\bar{\ell}_{i}, i=1,2$ (see Fig. 3). By construction, $\theta$ restricts to a diffeomorphism $W(\mathbb{R}) \approx W_{1}(\mathbb{R})$, the dual graph of the proper transform of $\tau^{-1}(B)$ by $\theta_{1}$ is depicted in Fig. 4 .

Now let $\theta_{2}: W_{1} \rightarrow W_{2}$ be the real birational map obtained by first blowing-up the point $E_{1} \cap E_{3}$ with exceptional divisor $D_{1}$ and then contracting successively the proper transforms of $B, E_{3}$ and $E_{2}$. By counting the number of points blown-up and the number of curves contracted during the whole process, we infer that the Picard rank of $W_{2}$ is equal to 2 . So $W_{2}$ is a Hirzebruch surface in which the proper transforms of $D_{1}$ and $E_{1}$ are respectively a fiber and a section with self-intersection 1 of a $\mathbb{P}^{1}$ bundle structure on $W_{2}$. It follows that $W_{2} \simeq \mathbb{F}_{1}$ and that the proper transform of $D_{1}$ is a fiber of the unique $\mathbb{P}^{1}$-bundle structure $\pi_{1}: \mathbb{F}_{1} \rightarrow \mathbb{C P}^{1}$. The restriction of $\theta_{2} \circ \theta_{1}$ to $S$ is a real birational map $f: S \rightarrow \mathbb{F}_{1} \backslash\left(E_{1} \cup D_{1}\right) \simeq \mathbb{C}^{2}$ inducing a diffeomorphism $S(\mathbb{R}) \approx \mathbb{R}^{2}$.

II. For $S^{\prime}$, the construction is very similar although of a slightly different flavour. We first observe that the proper transform in the real minimal resolution $\tau^{\prime}: W^{\prime} \rightarrow V^{\prime}$ of 


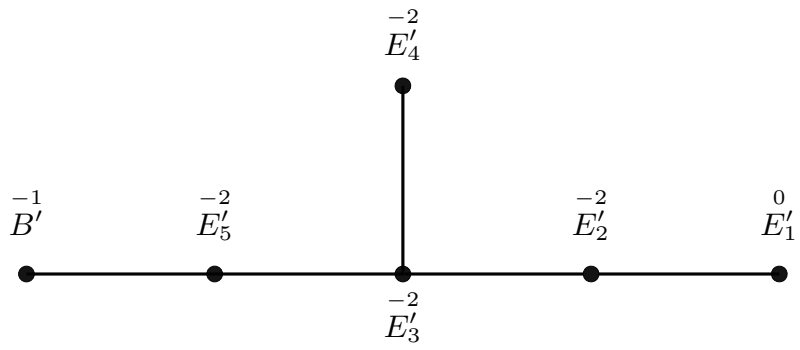

Fig. 5 Dual graph of the proper transform of $\left(\tau^{\prime}\right)^{-1}\left(B^{\prime}\right)$ by $\theta_{1}^{\prime}$

the pair $\left(V^{\prime}, B^{\prime}\right)$ of a pair of general nonreal complex conjugate hyperplane sections $\left(\ell^{\prime}, \bar{\ell}^{\prime}\right)$ of $V^{\prime}=\left\{x^{2} z-y^{3}+x t^{2}=0\right\}$ of the form $V^{\prime} \cap\{y-a t=0\}$ and $V^{\prime} \cap\{y-\bar{a} t=$ $0\}, a \in \mathbb{C} \backslash \mathbb{R}$, consists of a pair of complex conjugate rational 0 -curves intersecting the proper transform of $B$ transversally in a pair of nonreal complex conjugate points $\left(q^{\prime}, \bar{q}^{\prime}\right)$. For every such pair $\left(\ell^{\prime}, \bar{\ell}^{\prime}\right)$, the real birational map $\theta_{1}^{\prime}: W^{\prime} \rightarrow W_{1}^{\prime}$ consisting of the blow-up of $q^{\prime}$ and $\bar{q}^{\prime}$ followed by the contraction of the proper transforms of $\ell^{\prime}$ and $\bar{\ell}^{\prime}$ restricts to a diffeomorphism $W^{\prime}(\mathbb{R}) \approx W_{1}^{\prime}(\mathbb{R})$. The dual graph of the proper transform of $\left(\tau^{\prime}\right)^{-1}\left(B^{\prime}\right)$ by $\theta_{1}^{\prime}$ is depicted in Fig. 5 .

Letting $\theta_{2}^{\prime}: W_{1}^{\prime} \rightarrow W_{2}^{\prime}$ be the real birational morphism consisting of the successive contractions of $B^{\prime}, E_{5}^{\prime}, E_{3}^{\prime}$ and $E_{4}^{\prime}$, the resulting surface $W_{2}^{\prime}$ is isomorphic to $\mathbb{C P}^{1} \times \mathbb{C P}^{1}$ in which the proper transforms of $E_{1}^{\prime}$ and $E_{2}^{\prime}$ are fibers say of the first and second projection respectively. The restriction of $\theta_{2}^{\prime} \circ \theta_{1}^{\prime}$ to $S^{\prime}$ is a real birational map $f^{\prime}: S^{\prime} \rightarrow$ $\mathbb{C P}^{1} \times \mathbb{C P}^{1} \backslash\left(E_{1}^{\prime} \cup E_{2}^{\prime}\right) \simeq \mathbb{C}^{2}$ inducing a diffeomorphism $S^{\prime}(\mathbb{R}) \approx \mathbb{R}^{2}$.

\section{Fake planes of Kodaira dimension 0}

By virtue of [17, Theorem 4.7.1(1), p. 244], there is no smooth complex $\mathbb{Z}$-acyclic surface of Kodaira dimension 0. Smooth complex $\mathbb{Q}$-acyclic surfaces of Kodaira dimension 0 do exist, and are completely classified after the work of Fujita and Kojima (see also [17, Chapter 3, Section 4]). In this section, we present certain families of fake planes of Kodaira dimension 0 , some of which being $\mathbb{R}$-biregularly birationally equivalent to $\mathbb{C}^{2}$.

\subsection{Real models of Fujita's $H[-k, k]$ surfaces}

In the Hirzebruch surface $\pi_{2 p}: \mathbb{F}_{2 p} \rightarrow \mathbb{C P}^{1}, p \geqslant 1$, with negative section $C_{0} \simeq \mathbb{C P}^{1}$ of self-intersection $-2 p$, we choose a real section $C_{1} \sim C_{0}+(2 p+1) f$, where $f$ is a general real fiber of $\pi_{2 p}$, and a pair $(\ell, \bar{\ell})$ of nonreal complex conjugate fibers of $\pi_{2 p}$. Note that since the unique intersection point of $C_{1}$ and $C_{0}$ is real, neither $\ell$ nor $\bar{\ell}$ passes through it. Now we let $\tau: V \rightarrow \mathbb{F}_{2 p}$ be the smooth real projective surface obtained from $\mathbb{F}_{2 p}$ by first blowing-up the pair of nonreal complex conjugate points $q_{1}=C_{1} \cap \ell$ and $\bar{q}_{1}=C_{1} \cap \bar{\ell}$ with respective exceptional divisors $E$ and $\bar{E}$ and then blowing-up the pair of nonreal complex conjugate points $q_{1}^{\prime}=\ell \cap E$ and $\bar{q}_{1}^{\prime}=\bar{\ell} \cap \bar{E}$ with respective 


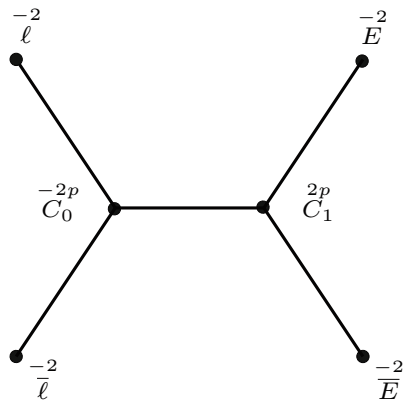

Fig. 6 Dual graph of $B$

exceptional divisors $F$ and $\bar{F}$. The dual graph of $B=C_{0} \cup C_{1} \cup \ell \cup \bar{\ell} \cup E \cup \bar{E}$, where we identified each curve with its proper transform in $V$, is a tree depicted in Fig. 6 .

Since $B$ is a real curve, $H[-2 p, 2 p]=V \backslash B$ is a smooth real quasi-projective surface. The abelian group $\mathbb{Z}\langle B\rangle$ is freely generated by the classes of the irreducible curves $C_{0}, C_{1}, \ell, \bar{\ell}, E$ and $\bar{E}$ while the divisor class group $\mathrm{Cl}(V)$ of $V$ is freely generated by $C_{0}, f, E, \bar{E}, F, \bar{F}$. Using the relations

$$
\left\{\begin{array}{l}
\tau^{*} C_{1}=C_{1}+E+\bar{E}+F+\bar{F} \sim C_{0}+(2 p+1) f, \\
\tau^{*} \ell=\ell+E+2 F \sim f \\
\tau^{*} \bar{\ell}=\bar{\ell}+\bar{E}+2 \bar{F} \sim f
\end{array}\right.
$$

in $\mathrm{Cl}(V)$, we find that the homomorphism $j_{*}: \mathbb{Z}\langle B\rangle \rightarrow \mathrm{Cl}(V)$ induced by the inclusion $B \hookrightarrow V$ is represented by the matrix

$$
\left(\begin{array}{cccccc}
1 & 1 & 0 & 0 & 0 & 0 \\
0 & 2 p+1 & 1 & 1 & 0 & 0 \\
0 & -1 & -1 & 0 & 1 & 0 \\
0 & -1 & 0 & -1 & 0 & 1 \\
0 & -1 & -2 & 0 & 0 & 0 \\
0 & -1 & 0 & -2 & 0 & 0
\end{array}\right) .
$$

It follows from Theorem 2.1 (a) and Remark 2.2 that $S=H[-2 p, 2 p]$ is $\mathbb{Q}$-acyclic, with $H_{1}(S ; \mathbb{Z}) \simeq \mathbb{Z}_{8 p}$. On the other hand, the real locus of $B$ is homeomorphic to a wedge of two circles, hence is in particular nonempty. The Galois cohomology group $H^{2}(G, \mathbb{Z}\langle B\rangle)$ is freely generated by the classes of $C_{0}$ and $C_{1}$ while $H^{2}(G, \mathrm{Cl}(V))$ is freely generated by the classes of $C_{0}$ and $f$. The relations above imply that the matrix of the homomorphism $H^{2}\left(j_{*}\right): H^{2}(G, \mathbb{Z}\langle B\rangle) \rightarrow H^{2}(G, \mathrm{Cl}(V))$ with respect to these bases is the identity. So $H[-2 p, 2 p]$ is a real plane by virtue of Theorem 2.1 (b).

Proposition 4.1 For every $p \geqslant 1$, the surface $H[-2 p, 2 p]$ is a $\mathbb{Q}$-acyclic fake plane of Kodaira dimension $0, \mathbb{R}$-biregularly birationally equivalent to $\mathbb{C}^{2}$.

Proof The fact that $S=H[-2 p, 2 p]$ if a fake plane follows from the discussion above. Since $K_{\mathbb{F}_{2 p}} \sim-2 C_{0}-(2 p+2) f$, we deduce from the ramification formula 
for $\tau: V \rightarrow \mathbb{F}_{2 p}$ and the relations in $\mathrm{Cl}(V)$ indicated above that

$$
\begin{aligned}
& K_{V}+B \sim-2 C_{0}-(2 p+2) f+E+\bar{E}+2 F+2 \bar{F} \\
&+\left(C_{0}+C_{1}+E+\bar{E}+\ell+\bar{\ell}\right) \\
& \sim f-F-\bar{F}
\end{aligned}
$$

hence that

$$
2\left(K_{V}+B\right) \sim 2(f-F-\bar{F}) \sim(f-2 F)+(f-2 \bar{F}) \sim \ell+E+\bar{\ell}+\bar{E} .
$$

So the linear system $\left|2\left(K_{V}+B\right)\right|$ is nonempty, which implies that $\kappa(S) \geqslant 0$, and since the intersection matrix of $\ell+E+\bar{\ell}+\bar{E}$ is negative definite it follows that $\kappa(S)=0$. That $S$ is $\mathbb{R}$-biregularly birationally equivalent to $\mathbb{C}^{2}$ can be seen as follows. First since the real morphism $\tau: V \rightarrow \mathbb{F}_{2 p}$ consists of blow-ups of nonreal points only, its restriction to $S$ is a birational morphism $S \rightarrow S^{\prime}=\mathbb{F}_{2 p} \backslash\left(C_{0} \cup C_{1}\right)$ inducing a diffeomorphism $S(\mathbb{R}) \approx S^{\prime}(\mathbb{R})$. The smooth real affine surface $S^{\prime}=\mathbb{F}_{2 p} \backslash\left(C_{0} \cup C_{1}\right)$ admits a real $\mathbb{A}^{1}$ fibration $\rho: S^{\prime} \rightarrow \mathbb{C}$ induced by the restriction of the pencil $\mathbb{F}_{2 p} \rightarrow \mathbb{C P}^{1}$ generated by the linearly equivalent divisors $C_{1}$ and $C_{0}+(2 p+1) F_{0}$, where $F_{0} \simeq \mathbb{C P}^{1}$ denotes the fiber of $\pi_{2 p}$ over the real point $\pi_{2 p}\left(C_{0} \cap C_{1}\right) \in \mathbb{C P}^{1}$. This fibration has a unique degenerate fiber of multiplicity $2 p+1$ consisting of intersection of $F_{0}$ with $S^{\prime}$, and so $S^{\prime}$ is $\mathbb{R}$-biregularly equivalent to $\mathbb{C}^{2}$ by virtue of Theorem 3.3. So $S(\mathbb{R})$ is birationally diffeomorphic to $\mathbb{R}^{2}$.

\subsection{The exceptional fake plane $Y(3,3,3)$ (see also [8, Section 5.1.1])}

Let $D$ be the union of four general real lines $\ell_{i} \simeq \mathbb{C P}^{1}, i=0,1,2,3$, in $\mathbb{C P}^{2}$ and let $\tau: V \rightarrow \mathbb{C P}^{2}$ be the real projective surface obtained by first blowing-up the points $p_{i j}=\ell_{i} \cap \ell_{j}$ with exceptional divisors $E_{i j}, i, j=1,2,3, i \neq j$, and then blowing-up the points $\ell_{1} \cap E_{12}, \ell_{2} \cap E_{23}$ and $\ell_{3} \cap E_{13}$ with respective exceptional divisors $E_{1}, E_{2}$ and $E_{3}$. We let $B=\ell_{0} \cup \ell_{1} \cup \ell_{2} \cup \ell_{3} \cup E_{12} \cup E_{23} \cup E_{13}$. The dual graphs of $D$, its total transform $\tau^{-1}(D)$ in $V$ and $B$ are depicted in Fig. 7.

Since $B$ is a real curve, $Y(3,3,3)=V \backslash B$ is a smooth real quasi-projective surface. The divisor class group $\mathrm{Cl}(V)$ of $V$ is freely generated by $\ell_{0}, E_{12}, E_{23}, E_{13}, E_{1}, E_{2}$ and $E_{3}$ and using the relations

$$
\left\{\begin{array}{l}
\tau^{*} \ell_{1}=\ell_{1}+E_{12}+E_{13}+2 E_{1}+E_{3} \sim \ell_{0}, \\
\tau^{*} \ell_{2}=\ell_{2}+E_{12}+E_{23}+E_{1}+2 E_{2} \sim \ell_{0}, \\
\tau^{*} \ell_{3}=\ell_{3}+E_{13}+E_{23}+E_{2}+2 E_{3} \sim \ell_{0}
\end{array}\right.
$$

in $\mathrm{Cl}(V)$ together with the ramification formula for $\tau$, we find that

$$
\begin{aligned}
3\left(K_{V}+B\right) & \sim 3 \ell_{0}-3\left(E_{1}+E_{2}+E_{3}\right) \\
& \sim\left(\ell_{1}+\ell_{2}+\ell_{3}\right)+2\left(E_{12}+E_{13}+E_{23}\right) .
\end{aligned}
$$




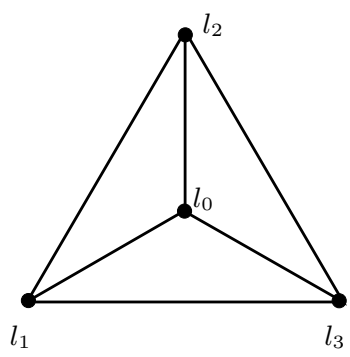

D

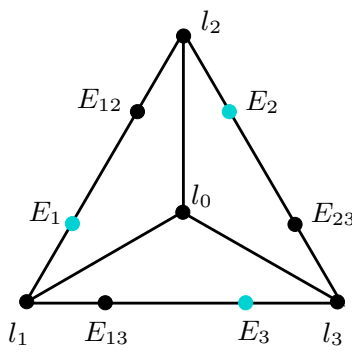

$\tau^{-1}(D)$

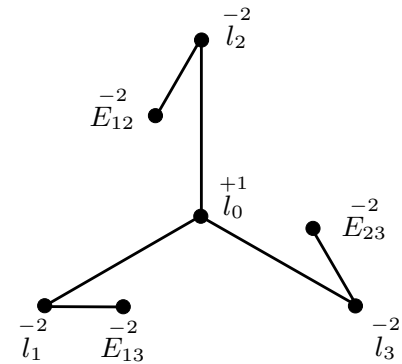

$B$

Fig. 7 Construction of $Y(3,3,3)$

So $\kappa(Y(3,3,3)) \geqslant 0$ and since the intersection matrix of the divisor on the right hand is negative definite, it follows that $\kappa(Y(3,3,3))=0$. The abelian group $\mathbb{Z}\langle B\rangle$ is freely generated by the classes of the irreducible curves $\ell_{i}, i=0, \ldots, 3, E_{12}, E_{23}$ and $E_{13}$ and using the above relations, we find that the homomorphism $j_{*}: \mathbb{Z}\langle B\rangle \rightarrow \mathrm{Cl}(V)$ induced by the inclusion $B \hookrightarrow V$ is represented by the matrix

$$
M=\left(\begin{array}{ccccccc}
1 & 1 & 1 & 1 & 0 & 0 & 0 \\
0 & -1 & -1 & 0 & 1 & 0 & 0 \\
0 & 0 & -1 & -1 & 0 & 1 & 0 \\
0 & -1 & 0 & -1 & 0 & 0 & 1 \\
0 & -2 & -1 & 0 & 0 & 0 & 0 \\
0 & 0 & -2 & -1 & 0 & 0 & 0 \\
0 & -1 & 0 & -2 & 0 & 0 & 0
\end{array}\right)
$$

which has determinant det $M=-9$. Furthermore, since $\tau: V \rightarrow \mathbb{C P}^{2}$ consists of blow-ups of real points only, $H^{2}(G, \mathbb{Z}\langle B\rangle) \simeq \mathbb{Z}\langle B\rangle \otimes_{\mathbb{Z}} \mathbb{Z}_{2}, H^{2}(G, \mathrm{Cl}(V)) \simeq$ $\mathrm{Cl}(V) \otimes_{\mathbb{Z}} \mathbb{Z}_{2}$ and the homomorphism $H^{2}\left(j_{*}\right)$ is represented by the reduction modulo 2 of $M$. It follows from Theorem 2.1 that $Y(3,3,3)$ is a $\mathbb{Q}$-acyclic fake plane, with $H_{1}(Y(3,3,3) ; \mathbb{Z}) \simeq \mathbb{Z}_{9}$.

Question 4.2 Is the real locus of $Y(3,3,3)$ birationally diffeomorphic to $\mathbb{R}^{2}$ ?

\section{Fake planes of Kodaira dimension 1}

In this section, we first recall basic results on the classification of $\mathbb{Z}$-acyclic fake planes of Kodaira dimension 1 . We then present examples of contractible and $\mathbb{Q}$-acyclic fake planes of Kodaira dimension 1 arising from rational real cuspidal quartics in $\mathbb{C P}^{2}$. We conclude this section with the construction of families of $\mathbb{Q}$-acyclic fake planes $\mathbb{R}$-biregularly birationally equivalent to $\mathbb{C}^{2}$. 


\subsection{Elements of classification of $\mathbb{Z}$-acyclic fake planes of Kodaira dimension 1}

Smooth complex $\mathbb{Z}$-acyclic surfaces of Kodaira dimension 1 have been classified by Gurjar and Miyanishi [11] and tom Dieck and Petrie [4]. We recall from [8] the following construction, which provides the real counter-part of this classification.

\subsubsection{General blow-up construction}

Let $D \subset \mathbb{C P}^{2}$ be the union of a collection $E_{0,0}, \ldots, E_{n, 0} \simeq \mathbb{C P}^{1}$ of $n+1 \geqslant 3$ real lines intersecting in a same point $x$ and a general real line $C_{1} \simeq \mathbb{C P}^{1}$. For every $i=$ $1, \ldots, n$, we choose a pair of coprime integers $1 \leqslant \mu_{i,-}<\mu_{i,+}$ in such a way that for $v_{-}={ }^{t}\left(\mu_{1,-}, \ldots, \mu_{n,-}\right) \in \mathcal{M}_{n, 1}(\mathbb{Z})$ and $\Delta_{+}=\operatorname{diag}\left(\mu_{1,+}, \ldots, \mu_{n,+}\right) \in \mathcal{M}_{n, n}(\mathbb{Z})$, the following two conditions are satisfied:

- $\eta=n-1-\sum_{i=1}^{n} 1 / \mu_{i,+}>0$,

- The matrix $\mathcal{N}=\left(\begin{array}{cc}-1 & -1 \\ v_{-} & \Delta_{+}\end{array}\right)$belongs to $\mathrm{GL}_{n+1}(\mathbb{Z})$.

Then we let $\tau: V \rightarrow \mathbb{C P}^{2}$ be the smooth real projective surface obtained by the following blow-up procedure:

1) We first blow-up $x$ with exceptional divisor $C_{0} \simeq \mathbb{C P}^{1}$. The resulting surface is isomorphic to the Hirzebruch surface $\pi_{1}: \mathbb{F}_{1} \rightarrow \mathbb{C P}^{1}$ with $C_{0}$ as the negative section of $\pi_{1}$, the proper transforms of $E_{0,0}, \ldots, E_{n, 0}$ are fibers of $\pi_{1}$ while the strict transform of $C_{1}$ is a section of $\pi_{1}$ disjoint from $C_{0}$.

2) Then for every $i=1, \ldots, n$, we perform a sequence of blow-up of real points, starting with the blow-up of $p_{i}=C_{1} \cap E_{i, 0}$ in such a way that the following two conditions are satisfied: a) the inverse image of $p_{i}$ is a chain of $\mathbb{C P}^{1}$ containing a unique $(-1)$-curve $A\left(p_{i}\right)$ and b) the coefficients of $A\left(p_{i}\right)$ in the total transform of $C_{1}$ and $E_{i, 0}$ are equal to $\mu_{i,-}$ and $\mu_{i,+}$ respectively. Recall [8, Example 1.3] that letting $x_{i,-}$ and $x_{i,+}$ be local defining equations for $C_{1}$ and $E_{i, 0}$ in a Zariski open neighborhood $U_{i}$ of $p_{i}$, such a blow-up sequence is obtained as the minimal resolution of the indeterminacies at $p_{i}$ of the rational map $x_{i,+}^{\mu_{i,+}} / x_{i,-}^{\mu_{i,-}}: U_{i} \rightarrow \mathbb{C P}^{1}$. We denote by $E_{i, 1}, \ldots, E_{i, r_{i}-1}, E_{i, r_{i}}=A\left(p_{i}\right)$ the corresponding exceptional divisors.

3) Finally, we perform a sequence of blow-ups starting with the blow-up of a real point $p_{0} \in E_{0,0} \backslash\left(C_{0} \cup C_{1}\right)$, with exceptional divisor $E_{0,1} \simeq \mathbb{C P}^{1}$ and continuing with a sequence of $r_{0}-1 \geqslant 0$ blow-ups of real points $p_{0, i} \in E_{0, i} \backslash E_{0, i-1}, i=1, \ldots, r_{0}-1$, with exceptional divisors $E_{0, i+1}$. We let $A\left(p_{0}\right)=E_{0, r_{0}}$.

The union $B$ of the proper transforms of $C_{0}, C_{1}$, and the divisors $E_{i, j}, i=$ $0, \ldots, n, j=0, \ldots, r_{i-1}$, is a real subtree of the total transform of $D$ by the soconstructed morphism $\tau: V \rightarrow \mathbb{C P}^{2}$. By virtue of [17, Lemma 4.5.3, p. 237] and the proof of [17, Theorem 4.6.1, p. 238], we have

$$
\begin{aligned}
K_{V}+B & \sim\left(\pi_{1} \circ \tau\right)^{*} K_{\mathbb{C P}^{1}}+\ell+\sum_{i=1}^{n}\left(\left(\pi_{1} \circ \tau\right)^{*} E_{i, 0}-A\left(p_{i}\right)\right) \\
& \sim(n-1) \ell-\sum_{i=1}^{n} A\left(p_{i}\right) \sim \eta \ell+N=P+N
\end{aligned}
$$




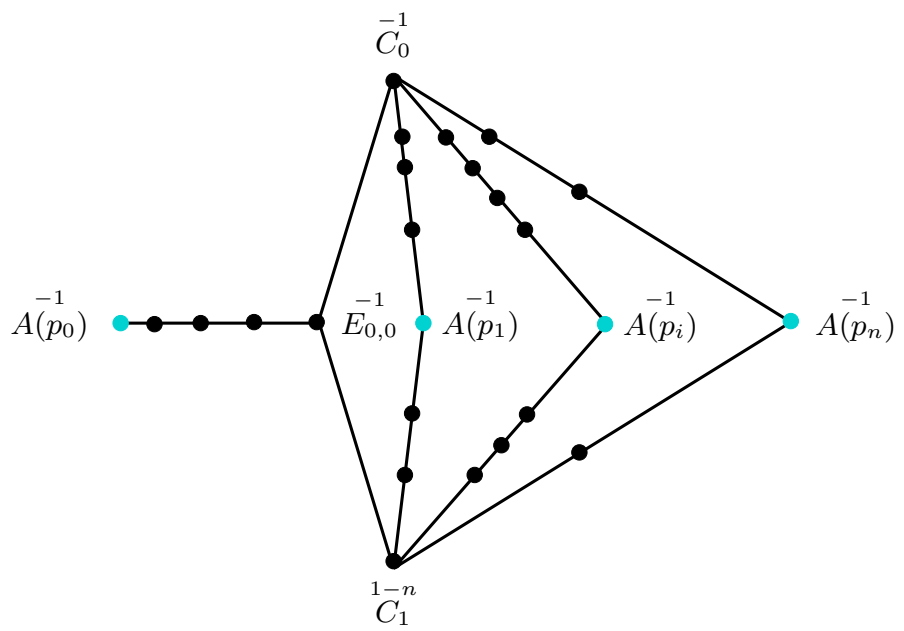

Fig. 8 Dual graph of $\tau^{-1}(D)$

where $\ell$ is the proper transform of a general real fiber of $\pi_{1}$, and $N$ is an effective $\mathbb{Q}$-divisor supported on $\sum_{i=1}^{n}\left(\left(\pi_{1} \circ \tau\right)^{*} E_{i, 0}\right)_{\text {red }}-A\left(p_{i}\right)$. So the intersection matrix of $N$ is negative definite and since $\eta>0$ by hypothesis, it follows that $P$ is nef. The surface $S=V \backslash B$ is thus a real surface of Kodaira dimension $\kappa(S)=1$. Note further that the morphism $\pi_{1} \circ \tau: V \rightarrow \mathbb{C P}^{1}$ coincides with that induced by the positive part of $K_{V}+B$ and that it restricts on $S$ to a fibration $S \rightarrow \mathbb{C P}^{1}$ with general fibers isomorphic to $\mathbb{C}^{*}$. We have the following classification result.

Theorem 5.1 ([8, Theorem 3.3]) Every $\mathbb{Z}$-acyclic fake plane $S$ of Kodaira dimension 1 is isomorphic to a surface constructed by the above procedure.

We do not know whether any of these surfaces is $\mathbb{R}$-biregularly birationally equivalent to $\mathbb{C}^{2}$.

\subsection{Fake planes obtained from rational real cuspidal quartic curves}

\subsubsection{First construction}

Let $D \subset \mathbb{C P}^{2}$ be a real smooth rational quartic with a unique cusp $p_{0}$ of multiplicity 3 and a unique flex $q$ at which $D$ and its tangent $T_{q}$ intersect with multiplicity 4 . For instance, $D$ is the zero locus of the homogeneous polynomial $z^{4}-x y^{3} \in \mathbb{C}[x, y, z]$, the corresponding points $p_{0}$ and $q$ being $[1: 0: 0]$ and $[0: 1: 0]$.

Let $\tau: V \rightarrow \mathbb{C P}^{2}$ be the blow-up of any real point $p$ of $D$ different from $p_{0}$ and $q$, say with exceptional divisor $E_{0,1}$. Let $E_{0,0}$ and $E_{1,0}$ be the proper transforms of $D$ and $T_{q}$ respectively in $V$ and let $B=E_{0,0} \cup E_{1,0}$. Then $S=V \backslash B$ is a contractible fake plane of Kodaira dimension 1 . Indeed, the fact that $S$ is a $\mathbb{Z}$-acyclic fake plane of Kodaira dimension 1 can be deduced directly from Theorem 5.1 by comparing the minimal $\log$-resolution $\beta: V^{\prime} \rightarrow V$ of the pair $(V, B)$ depicted in Fig. 10 below with Fig. 8 above for $n=2$, the curves $A\left(p_{1}\right)$ and $A\left(p_{2}\right)$ corresponding in this case to 


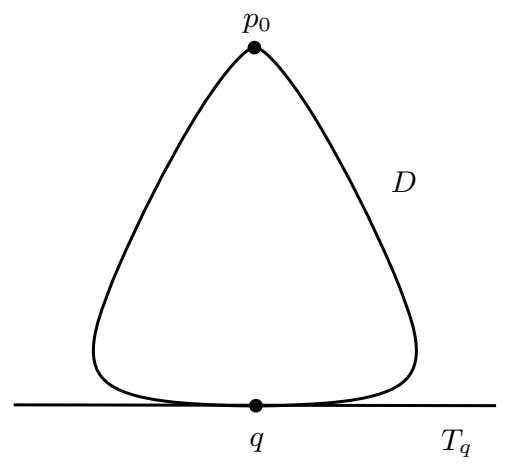

Fig. 9 Real ovoid quartic

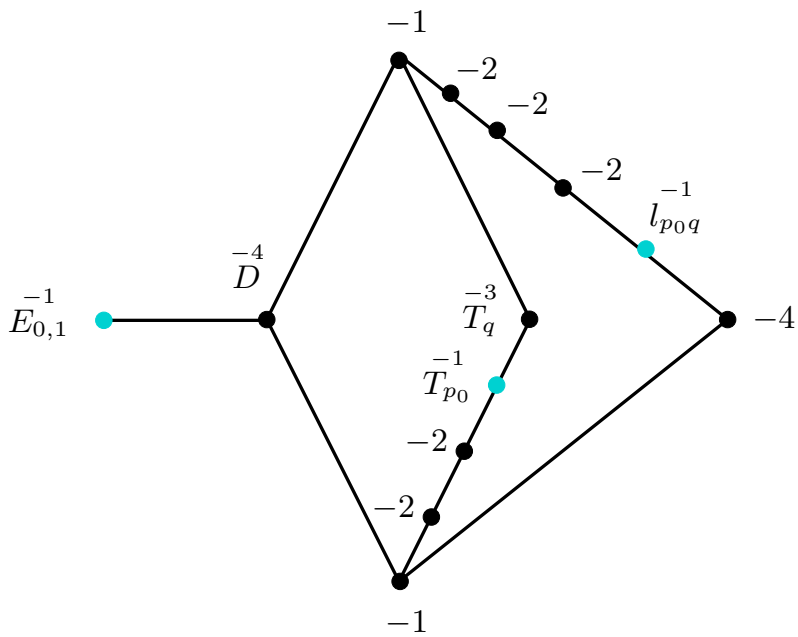

Fig. 10 Dual graph of the total transform of $B \cup T_{p_{0}} \cup \ell_{p_{0} q} \cup E_{0,1}$ in a minimal real log-resolution $\beta: V^{\prime} \rightarrow V$ of the pair $(V, B)$

the proper transforms of the tangent line $T_{p_{0}}$ to $B$ at $p_{0}$ and of the line $\ell_{p_{0} q}$ passing through $p_{0}$ and $q$ (Fig. 9).

Alternatively, the $\mathbb{Z}$-acyclicity follows from Theorem 2.2 via a computation similar to that in the previous section while the fact that $S(\mathbb{R}) \approx \mathbb{R}^{2}$ can be seen more directly as follows. Since $\tau: V \rightarrow \mathbb{C P}^{2}$ consists of the blow-up of a real point, $V(\mathbb{R})$ is a Klein bottle which we view as a circle bundle $\theta: V(\mathbb{R}) \rightarrow S^{1}$ with fibers equal to the real loci of the lines through $p$ in $\mathbb{C P}^{2}$. The sets $E_{0,1}(\mathbb{R})$ and $E_{1,0}(\mathbb{R})$ are two sections of $\theta$ which do no intersect each other. On the other hand, $E_{0,0}(\mathbb{R})$ is a connected closed curve which intersects $E_{0,1}(\mathbb{R})$ and $E_{1,0}(\mathbb{R})$ transversally in one point and at the point $q$ with multiplicity 4 . It follows that the homology classes of $E_{0,0}(\mathbb{R})$ and $E_{1,0}(\mathbb{R})$ in $H_{1}\left(V(\mathbb{R}) ; \mathbb{Z}_{2}\right)$ form a basis of this group. Since $B(\mathbb{R})$ is not empty, the long exact 
sequence of relative homology for the pair $(V(\mathbb{R}), B(\mathbb{R}))$ with $\mathbb{Z}_{2}$-coefficients implies that $S(\mathbb{R})$ is connected and $\mathbb{Z}_{2}$-acyclic, hence diffeomorphic to $\mathbb{R}^{2}$.

The contractibility of $S$ follows from the general classification of such surfaces given in [4]. It can also be seen directly as follows: since it is $\mathbb{Z}$-acyclic, it follows from the Hurewicz theorem that $S$ is contractible if and only if it is simply connected. Since $S \backslash E_{0,1} \simeq \mathbb{C P}^{2} \backslash\left(D \cup T_{q}\right)$ is the complement of a closed submanifold of real codimension 2 in $S, \pi_{1}(S)$ is a quotient of $\pi_{1}\left(\mathbb{C P}^{2} \backslash\left(D \cup T_{q}\right)\right)$. Furthermore, by virtue of $[22$, Lemma 2.3 (a) $]$, we have an exact sequence $0 \rightarrow \mathbb{Z} \cdot\langle\mu\rangle \rightarrow \pi_{1}\left(\mathbb{C P}^{2} \backslash(D \cup\right.$ $\left.\left.T_{q}\right)\right) \rightarrow \pi_{1}\left(\mathbb{C P}^{2} \backslash D\right) \rightarrow 0$ where $\mathbb{Z} \cdot\langle\mu\rangle$ is the free abelian group generated by a meridian of the line $T_{q}$. Since $\pi_{1}\left(\mathbb{C P}^{2} \backslash D\right)$ is abelian [6, Proposition 4.3, p. 130], so is $\pi_{1}\left(\mathbb{C P}^{2} \backslash\left(D \cup T_{q}\right)\right)$, implying in turn that $\pi_{1}(S)$ is abelian, hence trivial.

\subsubsection{Second construction}

Let $D \subset \mathbb{C P}^{2}$ be a real smooth rational quartic with a unique cusp $p_{0}$ of multiplicity 3 and a pair of real flexes $q_{1}$ and $q_{2}$ at which $D$ and its tangent $T_{q_{i}}$ intersect with multiplicity 3 . For instance, $D$ is the zero locus of the homogeneous polynomial $x^{3} y-z^{3} x+z^{4} \in \mathbb{C}[x, y, z]$, the corresponding points $p_{0}$ and $q_{1}$ and $q_{2}$ being $[0: 1: 0],[1: 0: 0]$ and $[3 / 2: 4 / 27: 1]$. Note that since a complex quartic with these properties is unique up to complex projective equivalence (see e.g. [19, pp. 135,146]), the construction below applies verbatim to any other real model of $D$. Let $\tau: V=$ $\mathbb{F}_{1} \rightarrow \mathbb{C P}^{2}$ be the blow-up of the intersection point $p$ of $T_{q_{1}}$ with $D$ different from $q_{1}$, say with exceptional divisor $F_{1}$. Since $p$ is real, $\tau$ is a real morphism and hence, the complement in $V$ of the proper transform $B$ of $D \cup T_{q_{1}}$ is a real surface. The relations $\tau^{*} D=D+F_{1}, \tau^{*} T_{q_{1}}=T_{q_{1}}+F_{1}$ in $\mathrm{Cl}(V)$ and $D \sim 4 T_{q_{1}}$ in $\mathrm{Cl}\left(\mathbb{C P}^{2}\right)$ imply that the homomorphism $j_{*}: \mathbb{Z}\langle B\rangle \rightarrow \mathrm{Cl}(V)$ induced by the inclusion $j: B \hookrightarrow V$ is represented in appropriate bases by a matrix of the form

$$
M=\left(\begin{array}{cc}
4 & 1 \\
-1 & -1
\end{array}\right) \text {. }
$$

Furthermore, $H^{2}(G, \mathbb{Z}\langle B\rangle) \simeq \mathbb{Z}\langle B\rangle \otimes_{\mathbb{Z}} \mathbb{Z}_{2}, H^{2}(G, \mathrm{Cl}(V)) \simeq \mathrm{Cl}(V) \otimes_{\mathbb{Z}} \mathbb{Z}_{2}$ as $\tau$ is the blow-up of a real point, and the homomorphism $H^{2}\left(j_{*}\right)$ is represented by the reduction modulo 2 of $M$. It follows from Theorem 2.1 and Remark 2.2 that $S$ is a $\mathbb{Q}$-acyclic fake plane, with $H_{1}(S ; \mathbb{Z}) \simeq \mathbb{Z}_{3}$ as det $M=-3$. In contrast with the $\mathbb{Z}$-acyclic fake planes considered in Sect. 5.1.1 which all admit a log-canonical real fibration over $\mathbb{C P}^{1}$ with general fibers isomorphic to $\mathbb{C}^{*}$, the surface $S$ just constructed admits a fibration of this type over the complex line $\mathbb{C}$. Namely, letting $\ell_{p_{0} q_{1}}=\{z=0\}$ and $\ell_{p_{0} p}=\{z-x=0\}$ be the lines through $p_{0}, q_{1}$ and the line through $p_{0}, p$ respectively, the divisors $D, 3 T_{p_{0}}+T_{q_{1}}=\left\{x^{3} y=0\right\}$ and $3 \ell_{p_{0} q_{1}}+\ell_{p_{0} p}=\left\{z^{3}(z-x)=0\right\}$ in $\mathbb{C P}^{2}$ generate a real pencil $V \rightarrow \mathbb{C P}^{1}$ containing $D=\left\{x^{3} y+z^{3}(z-x)=0\right\}$ as a member and whose lift in the minimal real log-resolution $\beta: \widetilde{V} \rightarrow V$ of the pair $(V, B)$ coincides with the $\mathbb{P}^{1}$-fibration $\widetilde{V} \rightarrow \mathbb{C P}^{1}$ generated by the proper transform of $D$ (see Fig. 11). This fibration restricts on $S$ to a real morphism $S \rightarrow \mathbb{C}$ with general fibers isomorphic to $\mathbb{C}^{*}$ and two degenerate fibers: one consisting of the disjoint union 


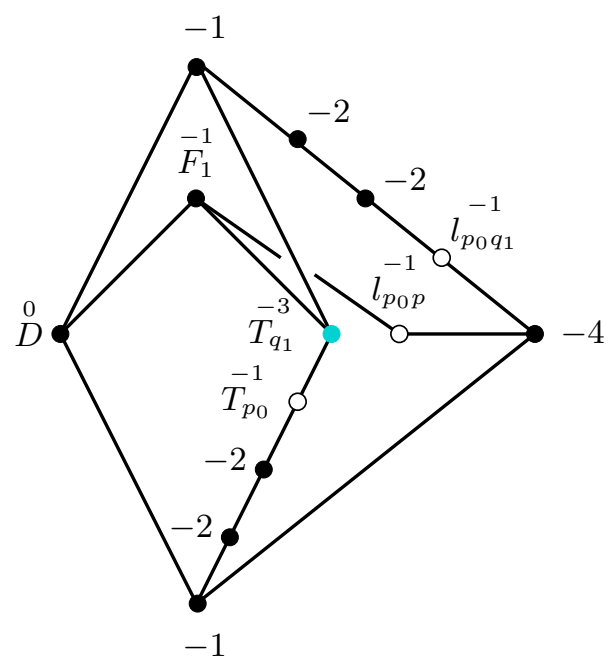

Fig. 11 Dual graph of $\beta^{-1}\left(B \cup T_{p_{0}} \cup \ell_{p_{0} q_{1}} \cup \ell_{p_{0} p}\right)$

of $\ell_{p_{0} p} \cap S \simeq \mathbb{C}$ and $\ell_{p_{0} q_{1}} \cap S \simeq \mathbb{C}^{*}$ the second component having multiplicity 3 , and a second one consisting of the intersection $T_{p_{0}} \cap S=\mathbb{C}^{*}$, also with multiplicity 3. The fact that $\kappa(S)=1$ then follows from a similar computation as in Sect. 5.1.1, see e.g. [17, Theorem 4.6.2, p. 237], which also renders the conclusion that $\widetilde{V} \rightarrow \mathbb{C P}^{1}$ coincides with the fibration induced by the positive part of $K_{\widetilde{V}}+\beta^{-1}(B)$.

\section{$5.3 \mathbb{Q}$-acyclic fake planes with real loci birationally diffeomorphic to $\mathbb{R}^{2}$ (see also [8, Remark 3.2])}

To construct families of $\mathbb{Q}$-acyclic fake planes $\mathbb{R}$-biregularly birationally isomorphic to $\mathbb{C}^{2}$, we start similarly as in Sect. 5.1.1 with the union $D \subset \mathbb{C P}^{2}$ of a real line $E_{0,0} \simeq \mathbb{C P}^{1}$, a collection $\left(E_{1,0}, \bar{E}_{1,0}\right), \ldots,\left(E_{m, 0}, \bar{E}_{m, 0}\right)$ of $m \geqslant 1$ distinct pairs of nonreal complex conjugated lines all meeting in a same real point $x$, and of a general real line $C_{1} \simeq \mathbb{C P}^{1}$. For every $i=1, \ldots, m$, we choose a pair of coprime integers $1 \leqslant v_{i,-}<v_{i,+}$ in such a way that for

$$
\left\{\begin{array}{l}
v_{-}={ }^{t}\left(v_{1,-}, \ldots, v_{m,-}, v_{1,-}, \ldots, v_{m,-}\right) \in \mathcal{M}_{2 m, 1}(\mathbb{Z}), \\
\Delta_{+}=\operatorname{diag}\left(v_{1,+}, \ldots, v_{m,+}, v_{1,+}, \ldots, v_{m,+}\right) \in \mathcal{M}_{2 m, 2 m}(\mathbb{Z})
\end{array}\right.
$$

the following two conditions are satisfied:

(a') $\eta=2 m-1-2 \sum_{k=1}^{m} 1 / v_{k,+}>0$,

(b') The matrix $\mathcal{M}=\left(\begin{array}{cc}-1 & -1 \\ v_{-} \Delta_{+}\end{array}\right)$belongs to $\mathrm{GL}_{2 m+1}(\mathbb{Q})$.

Then we let $\tau: V \rightarrow \mathbb{C P}^{2}$ be the real smooth projective surface obtained by the following blow-up procedure: 
1) We first blow-up $x$ with exceptional divisor $C_{0} \simeq \mathbb{C P}^{1}$. The resulting surface is isomorphic the Hirzebruch surface $\pi_{1}: \mathbb{F}_{1} \rightarrow \mathbb{C P}^{1}$ with $C_{0}$ as the negative section of $\pi_{1}$, the proper transform of $C_{1}$ is a section of $\pi_{1}$ disjoint from $C_{0}$, the proper transform of $E_{0,0}$ is a real fiber of $\pi_{1}$ while the proper transforms of the $E_{i, 0}$ and $\bar{E}_{i, 0}, i=1, \ldots, m$, are pairs of nonreal complex conjugate fibers of $\pi_{1}$.

2) Then for every $i=1, \ldots, m$, we perform a sequence of blow-ups of pairs of nonreal complex conjugate points, starting with the blow-up of the points $p_{i}=C_{1} \cap E_{i, 0}$ and $\bar{p}_{i}=C_{1} \cap \bar{E}_{i, 0}$ in such a way that the following two conditions are satisfied: a) the inverse images of $p_{i}$ and $\bar{p}_{i}$ are complex conjugate chains of $\mathbb{C P}^{1}$ each containing a unique $(-1)$-curve $A\left(p_{i}\right)$ and $A\left(\bar{p}_{i}\right)$ respectively and b) the coefficients of $A\left(p_{i}\right)$ (respectively of $A\left(\bar{p}_{i}\right)$ in the total transform of $C_{1}$ and $E_{i, 0}$ (respectively $\bar{E}_{i, 0}$ ) are equal to $v_{i,-}$ and $v_{i,+}$ respectively. We denote by $E_{i, 1}, \ldots, E_{i, r_{i}-1}, E_{i, r_{i}}=A\left(p_{i}\right)$ and $\bar{E}_{i, 1}, \ldots, \bar{E}_{i, r_{i}-1}, \bar{E}_{i, r_{i}}=A\left(\bar{p}_{i}\right)$ the corresponding exceptional divisors.

3) Finally, we perform a sequence of blow-ups starting with the blow-up of a real point $p_{0} \in E_{0,0} \backslash\left(C_{0} \cup C_{1}\right)$, with exceptional divisor $E_{0,1} \simeq \mathbb{C P}^{1}$ and continuing with a sequence of $r_{0}-1 \geqslant 0$ blow-ups of real points $p_{0, i} \in E_{0, i} \backslash E_{0, i-1}, i=1$, $\ldots, r_{0}-1$, with exceptional divisors $E_{0, i+1}$. We let $A\left(p_{0}\right)=E_{0, r_{0}}$.

The union $B$ of the proper transforms of $C_{0}, C_{1}$, and the divisors $E_{i, j}$ and $\bar{E}_{i, j}, i=0, \ldots, m, j=0, \ldots, r_{i-1}$, is a real subtree of the total transform $\widetilde{B}$ of $D$ by the so constructed morphism $\tau: V \rightarrow \mathbb{C P}^{2}$ (Fig. 12). Condition (a') guarantees by the same argument as in Sect. 5.1.1 that $S=V \backslash B$ is a real surface of Kodaira dimension 1 . The classes of the curves $C_{0}, E_{i, j}$ and $\bar{E}_{i, j}, i=1, \ldots, m, j=$ $0, \ldots, r_{i-1}, E_{0,0}, C_{1}-E_{0,0}, E_{0, i}-E_{0,0}, \bar{E}_{0, i}-E_{0,0}, i=1, \ldots, m$, form a basis of the free abelian group $\mathbb{Z}\langle B\rangle$ generated by the irreducible components of $B$ while $\mathrm{Cl}(V)$ is freely generated by the classes of $C_{0}, E_{i, j}$ and $\bar{E}_{i, j}, i=1, \ldots, m, j=$ $0, \ldots, r_{i-1}, E_{0,0}, A\left(p_{0}\right), A\left(p_{i}\right)$ and $A\left(\bar{p}_{i}\right), i=1, \ldots, m$. In these bases, the matrix of the homomorphism $j_{*}: \mathbb{Z}\langle B\rangle \rightarrow \mathrm{Cl}(V)$ induced by the inclusion takes the form

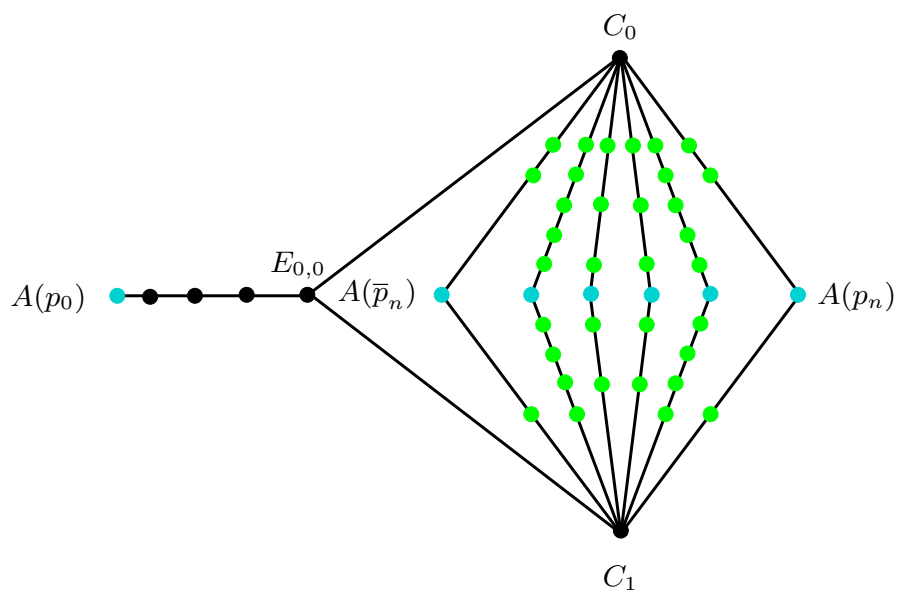

Fig. 12 Dual graph of $\tau^{-1}(D)$ 


$$
M=\left(\begin{array}{cc}
\operatorname{id}_{n} & * \\
0 & \mathcal{M}
\end{array}\right),
$$

where $n=2\left(\sum_{i=0}^{m} r_{i-1}+1\right)$. Since $\mathcal{M} \in \mathrm{GL}_{2 m+1}(\mathbb{Q})$ by hypothesis, we deduce from Theorem 2.1 (a) that $S$ is $\mathbb{Q}$-acyclic. But it is never $\mathbb{Z}$-acyclic as $v_{i,+} \geqslant 2$ for every $i=1, \ldots, m$. On the other hand, the real locus of $B$ is nonempty and the induced homomorphism $H^{2}\left(j_{*}\right): H^{2}(G ; \mathbb{Z}\langle B\rangle) \rightarrow H^{2}(G ; \mathrm{Cl}(V))$ is an isomorphism, implying that $S(\mathbb{R}) \approx \mathbb{R}^{2}$ by virtue of (b) in Theorem 2.1 .

Proposition 5.2 Every real surface $S$ as above is a $\mathbb{Q}$-acyclic fake plane of Kodaira dimension $1, \mathbb{R}$-biregularly birationally equivalent to $\mathbb{C}^{2}$.

Proof It remains to show that $S(\mathbb{R})$ is birationally diffeomorphic to $\mathbb{R}^{2}$. The real birational morphism $\tau: V \rightarrow \mathbb{C P}^{2}$ factors through the real projective surface $\tau^{\prime}: V^{\prime} \rightarrow \mathbb{C P}^{2}$ obtained from $\mathbb{C P}^{2}$ by blowing-up $x$ with exceptional divisor $C_{0}$ and then only performing the last sequence of blow-ups described in 3) above, the one starting with the blow-up of a real point $p_{0} \in E_{0,0} \backslash\left(C_{0} \cup C_{1}\right)$ (Fig. 13). Since the induced real birational morphism $\beta: V \rightarrow V^{\prime}$ consists of blow-ups of pairs of nonreal complex conjugate points only, it restricts to a birational diffeomorphism between the real loci of $V$ and $V^{\prime}$ respectively, mapping $S(\mathbb{R})$ diffeomorphically onto the real loci of the surface $S^{\prime}=V^{\prime} \backslash B^{\prime}$, where $B^{\prime}=C_{0} \cup C_{1} \cup \bigcup_{j=0}^{r_{0}-1} E_{0, j}$. A similar counting argument as in Sect. 3.2.2 shows that the surface obtained from $V^{\prime}$ by first blowing-up the real point $C_{1} \cap E_{0,0}$ with exceptional divisor $C$ and then contracting successively the proper transforms of $C_{0}, E_{0,0}, E_{0,1}, \ldots, E_{0, r_{0}-1}$ has Picard rank 2, hence is a Hirzebruch surface $\pi_{n}: \mathbb{F}_{n} \rightarrow \mathbb{C P}^{1}$ for some $n \geqslant 0$, in which the proper transforms of $C_{1}$ and $C$ are respectively a real fiber of $\pi_{n}$ and a real section of it, with self-intersection $r_{0}$. The so constructed real birational map $\theta: V^{\prime} \rightarrow \mathbb{F}_{n}$ induces an isomorphism between the real loci of $S^{\prime}$ and that of $\mathbb{F}_{n} \backslash\left(C \cup C_{1}\right)$, and the composition $\left.\theta \circ \beta\right|_{S}: S \rightarrow \mathbb{F}_{n} \backslash\left(C \cup C_{1}\right) \simeq \mathbb{C}^{2}$ is the desired $\mathbb{R}$-biregular birational isomorphism.
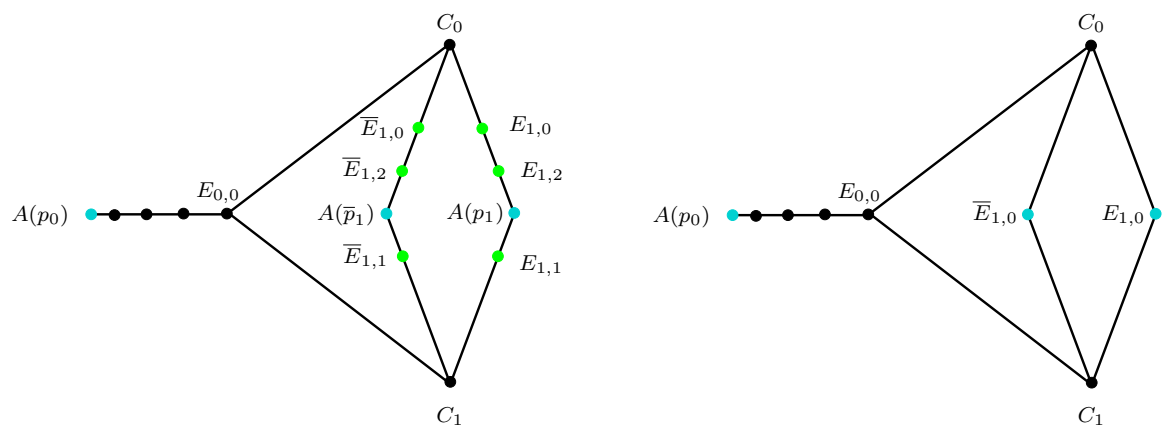

Fig. 13 Dual graphs of $\tau^{-1}(D)$ and $\tau^{\prime-1}(D)$ in the case $m=1, r_{1}=3,\left(v_{1,-}, v_{1,+}\right)=(2,3)$ 


\section{Fake planes of general type}

By virtue of [21], the complement $S$ of an irreducible rational cuspidal curve $B \subset \mathbb{C P}^{2}$ is a smooth affine surface of general type as soon as $B$ has at least three cusps. Such a surface $S$ is $\mathbb{Q}$-acyclic by virtue of Theorem 2.1 (a), with $H_{1}(S ; \mathbb{Z}) \simeq \mathbb{Z}_{d}$, where $d \geqslant 3$ denotes the degree of $B$. If in addition $B$ is a real curve with nonempty real locus, then Theorem 2.1 (b) implies that $S$ is a real plane if and only if $d$ is odd. This can be seen more directly as follows: when nonempty $B(\mathbb{R})$ is homeomorphic to a simple closed curve in $\mathbb{R} P^{2}$, whose homotopy class is either trivial if $d$ is even or equal to a generator of $\pi_{1}\left(\mathbb{R P}^{2}\right)$ if $d$ is odd. So $S(\mathbb{R})=\mathbb{R} \mathbb{P}^{2} \backslash B(\mathbb{R})$ is either diffeomorphic to the disjoint union of $\mathbb{R}^{2}$ with an open Möbius band in the first case or to $\mathbb{R}^{2}$ in the second case.

In this section, we first present different examples of contractible fake planes constructed from arrangements of lines and rational real cuspidal quartics in $\mathbb{C P}^{2}$ by the so-called cutting-cycle construction of tom Dieck and Petrie [5]. Then we construct an infinite family of $\mathbb{Z}$-acyclic fake planes of general type whose real loci are all birationally diffeomorphic to $\mathbb{R}^{2}$.

\subsection{Fake planes of general type obtained from rational real cuspidal quartic curves}

\subsubsection{First construction}

We consider again as in Sect. 5.2.2 a real smooth rational quartic $D_{1} \subset \mathbb{C P}^{2}$ with a unique cusp $p_{1,0}$ of multiplicity 3 and a pair of real flexes $q_{1,1}$ and $q_{1,2}$ at which $D_{1}$ and its tangent $T_{q_{i, 1}}\left(D_{1}\right)$ intersect with multiplicity 3 . We let $\tau_{1}: V_{1} \rightarrow \mathbb{C P}^{2}$ be the real projective surface obtained from $\mathbb{C P}^{2}$ by first blowing-up the intersection point $p_{1}$ of $T_{q_{1,1}}\left(D_{1}\right)$ with $D_{1}$ different from $q_{1,1}$ with exceptional divisor $F_{1,1}$, then blowing-up the intersection point of $F_{1,1}$ with the proper transform of $D_{1}$ with exceptional divisor $F_{1,2}$ and then blowing-up the intersection point of $F_{1,2}$ with the proper transform of $D_{1}$, with exceptional divisor $A\left(p_{1}\right)$. The complement in $V_{1}$ of $B_{1}=D_{1} \cup T_{q_{1,1}}\left(D_{1}\right) \cup$ $F_{1,1} \cup F_{1,2}$ is a smooth $\mathbb{Z}$-acyclic real surface $S_{1}$ with $S_{1}(\mathbb{R}) \approx \mathbb{R}^{2}$.

Indeed, by construction,

$$
\begin{aligned}
\tau_{1}^{*} D_{1} & =D_{1}+F_{1,1}+2 F_{1,2}+3 A\left(p_{1}\right), \\
\tau_{1}^{*} T_{q_{1,1}}\left(D_{1}\right) & =T_{q_{1,1}}\left(D_{1}\right)+F_{1,1}+F_{1,2}+A\left(p_{1}\right)
\end{aligned}
$$

so that using the relation $\tau_{1}^{*} D_{1} \sim 4 \tau_{1}^{*} T_{q_{1,1}}\left(D_{1}\right)$ in $\mathrm{Cl}\left(V_{1}\right)$, we obtain that in the bases of $\mathbb{Z}\left\langle B_{1}\right\rangle$ and $\mathrm{Cl}\left(V_{1}\right)$ given by the classes of the real curves, $T_{q_{1,1}}\left(D_{1}\right), F_{1,1}, F_{1,2}, D_{1}$ and $T_{q_{1,1}}\left(D_{1}\right), F_{1,1}, F_{1,2}, A\left(p_{1}\right)$ respectively, the homomorphism $j_{*}: \mathbb{Z}\left\langle B_{1}\right\rangle \rightarrow \mathrm{Cl}\left(V_{1}\right)$ induced by the inclusion $B_{1} \hookrightarrow V_{1}$ is represented by the matrix 


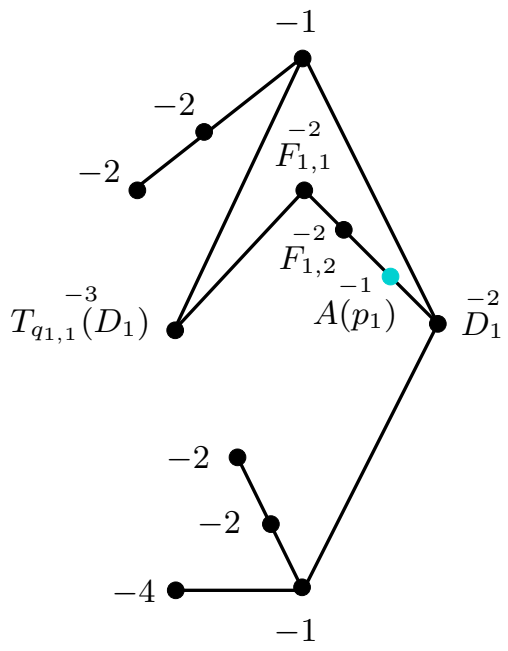

Fig. 14 Dual graph of the total transform of $B_{1} \cup A\left(p_{1}\right)$ in a minimal real log-resolution $\beta_{1}: V_{1}^{\prime} \rightarrow V_{1}$ of the pair $\left(V_{1}, B_{1}\right)$

$$
M=\left(\begin{array}{llll}
1 & 0 & 0 & 4 \\
0 & 1 & 0 & 3 \\
0 & 0 & 1 & 2 \\
0 & 0 & 0 & 1
\end{array}\right) \in \mathrm{GL}_{4}(\mathbb{Z})
$$

So $S_{1}$ is $\mathbb{Z}$-acyclic by Theorem 2.1 (a), and the same type of arguments as in Sect. 5.2.1 implies that $S_{1}$ is even contractible. On the other hand, since $\tau_{1}: V_{1} \rightarrow \mathbb{C P}^{2}$ consists of blow-ups of real points only, $H^{2}\left(j_{*}\right)$ is represented by the reduction modulo 2 of $M$, which is again invertible. Since $B_{1}(\mathbb{R})$ is nonempty, $S_{1}$ is a real plane by Theorem 2.1 (b).

\subsubsection{Second construction}

We let $D_{2} \subset \mathbb{C P}^{2}$ be a real rational ramphoid quartic, i.e. with a unique cusp $p_{2,0}$ with multiplicity sequence $(2,2,2)$, and three real flexes $q_{2,1}, q_{2,2}, q_{2,3}$ at which $D_{2}$ intersects its tangent $T_{q_{2, i}}\left(D_{2}\right)$ with multiplicity 3 . We let $\tau_{2}: V_{2} \rightarrow \mathbb{C P}^{2}$ be the real birational morphism obtained by blowing-up the intersection point $p_{2}$ of $D_{2}$ with $T_{q_{2,1}}\left(D_{2}\right)$ distinct from $q_{2,1}$, with exceptional divisor $F_{2,1}$, then blowing-up the intersection point of $F_{2,1}$ with the proper transform of $D_{2}$, with exceptional divisor $F_{2,2}$ and then blowing-up the intersection point of $F_{2,2}$ with the proper transform of $D_{2}$, with exceptional divisor $A\left(p_{2}\right)$. We let $S_{2}$ be the smooth real surface obtained as the complement of $B_{2}=D_{2} \cup T_{q_{2,1}}\left(D_{2}\right) \cup F_{2,1} \cup F_{2,2}$ in $V_{2}$.

The same computation as in Sect. 6.1.1 shows that $S_{2}$ is a contractible real plane. 


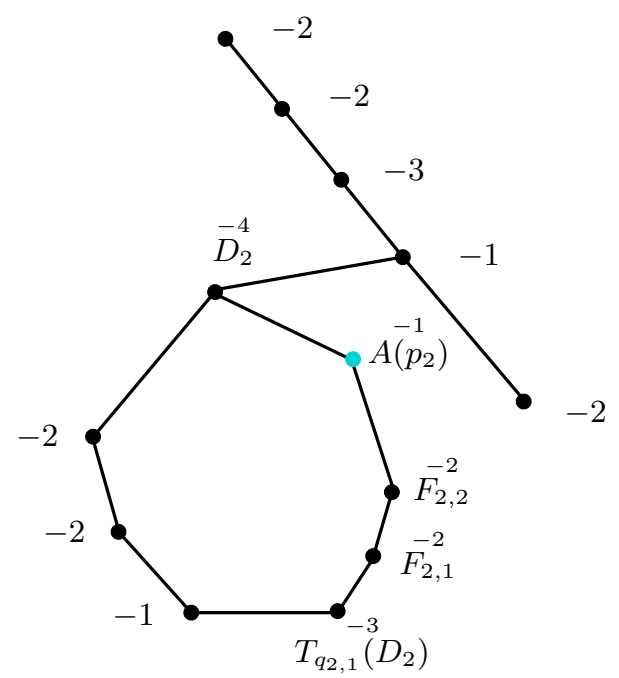

Fig. 15 Dual graph of the total transform of $B_{2} \cup A\left(p_{2}\right)$ in a minimal real log-resolution $\beta_{2}: V_{2}^{\prime} \rightarrow V_{2}$ of the pair $\left(V_{2}, B_{2}\right)$

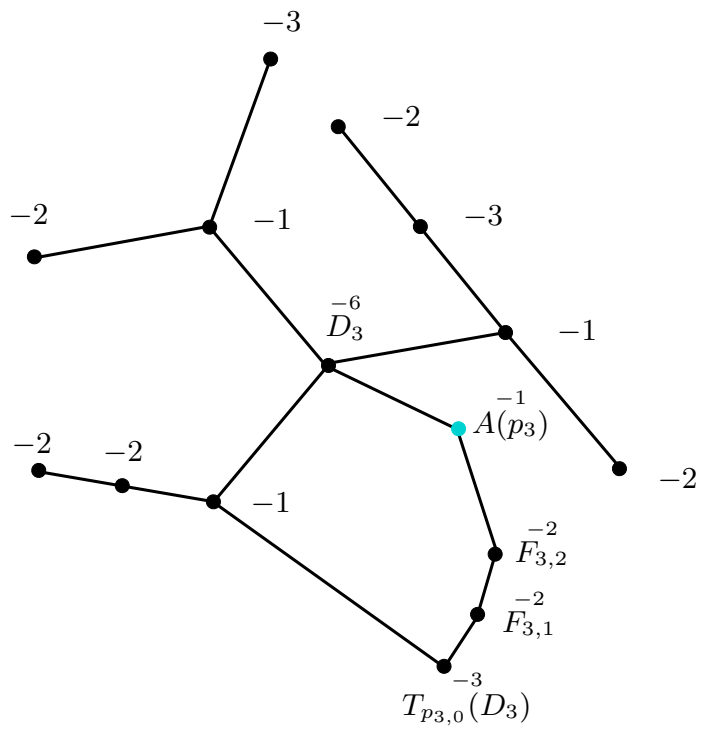

Fig. 16 Dual graph of the total transform of $B_{3} \cup A\left(p_{3}\right)$ in a minimal real log-resolution $\beta_{3}: V_{3}^{\prime} \rightarrow V_{3}$ of the pair $\left(V_{3}, B_{3}\right)$

\subsubsection{Third construction}

Here we start with a real rational bi-cuspidal quartic $D_{3} \subset \mathbb{C P}^{2}$, with two real cusps $p_{3, \infty}$ and $p_{3,0}$ with respective multiplicity sequences $(2,2)$ and $(2)$ such that $D_{3}$ intersects its tangent $T_{p_{3,0}}\left(D_{3}\right)$ with multiplicity 3 at $p_{3,0}$ and transversally at another 
real point $p_{3}$. We let $\tau_{3}: V_{3} \rightarrow \mathbb{C P}^{2}$ be the smooth real projective surface obtained from $\mathbb{C P}^{2}$ by first blowing-up $p_{3}$ with exceptional divisor $F_{3,1}$, then blowing-up the intersection point of $F_{3,1}$ with the proper transform of $D_{3}$ with exceptional divisor $F_{3,2}$ and then blowing-up the intersection point of $F_{3,2}$ with the proper transform of $D_{3}$ with exceptional divisor $A\left(p_{3}\right)$. We let $S_{3}$ be the smooth real surface obtained as the complement of $B_{3}=D_{3} \cup T_{p_{3,0}}\left(D_{3}\right) \cup F_{3,1} \cup F_{3,2}$ in $V_{3}$.

Similar arguments as in Sect. 6.1.1 imply that $S_{3}$ is a contractible real plane.

Proposition 6.1 The surfaces $S_{1}, S_{2}$ and $S_{3}$ constructed in Sects. 6.1.1, 6.1.2 and 6.1.3 are pairwise nonisomorphic contractible fake planes of general type.

Proof Letting $\beta_{i}: V_{i}^{\prime} \rightarrow V_{i}, i=1,2,3$, be the minimal real log-resolutions of the pair $\left(V_{i}, B_{i}\right)$ and $B_{i}^{\prime}=\beta_{i}^{-1}\left(B_{i}\right)$, the pairs $\left(V_{i}^{\prime}, B_{i}^{\prime}\right)$ are real minimal SNC-completion of $S_{i}$. Note that every irreducible component of $B_{i}^{\prime}$ has self-intersection $\leqslant-1$ and that every $(-1)$-curve in $B_{i}^{\prime}$ intersects three other irreducible components of $B_{i}^{\prime}$ (see Figs. 14, 15, 16). It follows that every birational map $\varphi:\left(V^{\prime \prime}, B^{\prime \prime}\right) \rightarrow\left(V_{i}^{\prime}, B_{i}^{\prime}\right)$ from another SNC-completion $\left(V^{\prime \prime}, B^{\prime \prime}\right)$ of $S_{i}$ restricting to an isomorphism between $V^{\prime \prime} \backslash B^{\prime \prime}$ and $V_{i}^{\prime} \backslash B_{i}^{\prime}$ is a morphism. Now if $S_{i}$ were isomorphic to $S_{j}$ for some $j \neq i$, then the birational map $\varphi:\left(V_{j}^{\prime}, B_{j}^{\prime}\right) \rightarrow\left(V_{i}^{\prime}, B_{i}^{\prime}\right)$, extending an isomorphism $S_{j} \stackrel{\sim}{\rightarrow} S_{i}$, would be an isomorphism mapping $B_{j}^{\prime}$ isomorphically onto $B_{i}^{\prime}$. In particular, the weighted dual graphs of $B_{j}^{\prime}$ and $B_{i}^{\prime}$ would be isomorphic, which is not the case. So $S_{1}, S_{2}$ and $S_{3}$ are pairwise nonisomorphic. It remains to show that they are all of general type. Since $\mathbb{C}^{2}$ is the only smooth $\mathbb{Z}$-acyclic real plane and since there is no $\mathbb{Z}$-acyclic real plane of Kodaira dimension 0 , to show that $\kappa\left(S_{i}\right)=2$, it is enough to check that $S_{i}$ is isomorphic neither to $\mathbb{C}^{2}$ nor to one of the surfaces described in Sect. 5.1.1. If $S_{i}$ were isomorphic to $\mathbb{C}^{2}$, then we would have a morphism $\varphi:\left(\mathbb{C P}^{2}, \ell\right) \rightarrow\left(V^{\prime}, B^{\prime}\right)$, where $\ell \simeq \mathbb{C P}^{1}$ is a real line, restricting to an isomorphism between $\mathbb{C P}^{2} \backslash \ell$ and $S_{i}$, which is impossible as $\ell^{2}>0$. Now suppose that $S_{i}$ is isomorphic to a surface obtained by the blow-up procedure $\tau^{\prime \prime}: V^{\prime \prime} \rightarrow \mathbb{C P}^{2}$ described in Sect. 5.1.1 and let $\left(V^{\prime \prime}, B^{\prime \prime}\right)$ be the corresponding SNC-completion with boundary $B^{\prime \prime}$ consisting of the proper transforms of $C_{0}, C_{1}$, and the divisors $E_{i, j}, i=0, \ldots, n, j=0, \ldots, r_{i-1}$. By construction, all irreducible components of $B^{\prime \prime}$ have self-intersection $\leqslant-1$ and $B^{\prime \prime}$ contains at most three $(-1)$-curves: the proper transform of $C_{0}$ and $E_{0,0}$, and the proper transform of $C_{1}$ if $n=2$, each of them intersecting at least three other irreducible components of $B^{\prime \prime}$, except in the case where $r_{0}=1$. Since the birational map $\varphi:\left(V^{\prime \prime}, B^{\prime \prime}\right) \rightarrow\left(V_{i}^{\prime}, B_{i}^{\prime}\right)$ induced by the isomorphism $V^{\prime \prime} \backslash B^{\prime \prime} \simeq S_{i} \simeq V^{\prime} \backslash B$ is a morphism, the structure of $B^{\prime \prime}$ implies that $\varphi$ is in fact an isomorphism of pairs, except possibly when $r_{0}=1$, where it can consist of the contraction of $E_{0,0}$ followed by an isomorphism of pairs. In both cases, we reach a contradiction by comparing the weighted dual graph of $B_{i}^{\prime}$ and the one of $B^{\prime \prime}$ or its image by the contraction of $E_{0,0}$ in the case where $r_{0}=1$. So $S_{i}$ is a fake plane of general type.

Question 6.2 Is the real locus of $S_{1}, S_{2}$ and $S_{3}$ birationally diffeomorphic to $\mathbb{R}^{2}$ ? 


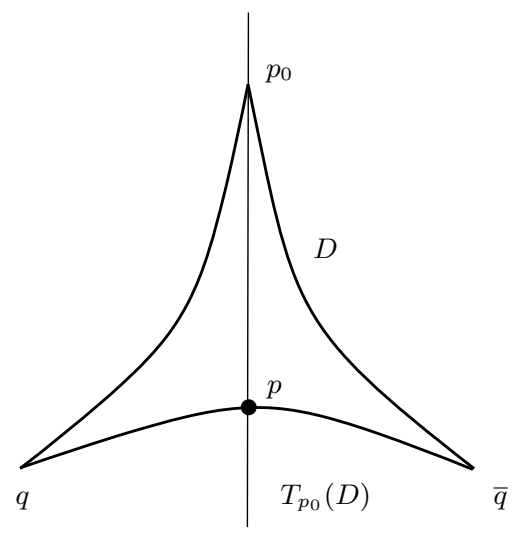

Fig. 17 Real tricuspidal quartic

\section{2 $\mathbb{Z}$-acyclic fake planes of general type $\mathbb{R}$-biregularly birationally equivalent to $\mathbb{C}^{2}$}

The projective dual $D \subset \mathbb{C P}^{2}$ of the nodal cubic $C$ with equation $(x-y)\left(x^{2}+y^{2}\right)-$ $x y z=0$ is a real rational quartic with three ordinary cusps: a real one $p_{0}$ corresponding to the real flex $[1: 1: 0]$ of $C$ and a pair of nonreal complex conjugate ones $q$ and $\bar{q}$ corresponding to the pair of nonreal conjugate flexes $[i: 1: 0]$ and $[-i: 1: 0]$ of $C$ (Fig. 17). The tangent $T_{p_{0}}(D)$ of $D$ at $p_{0}$ intersects $D$ with multiplicity 3 at $p_{0}$ and transversally at another real point $p$. Let $(\mu, \nu)$ be a pair of positive integers such that $4 v-\mu= \pm 1$ and let $\tau: V \rightarrow \mathbb{C P}^{2}$ be the real birational morphism obtained by first blowing-up $p$ with exceptional divisor $E_{1}$ and then, similarly as in 2) of Sect. 5.1.1, blowing-up a sequence of real points on the successive total transforms of $E_{1}$ in such a way that the following two conditions are satisfied: a) the inverse image of $p$ is a chain of curves isomorphic to $\mathbb{C P}^{1}$ containing a unique $(-1)$-curve $A(p)$ and b) the coefficients of $A(p)$ in the total transform of $D$ and $T_{p_{0}}(D)$ are equal to $\mu$ and $v$ respectively. We denote the corresponding exceptional divisors by $E_{1}, \ldots, E_{r-1}, E_{r}=A(p)$ and let $B=D \cup T_{p_{0}}(D) \cup \bigcup_{i=1}^{r-1} E_{i}$. The weighted dual graph of the total transform of $B \cup A(p)$ in a real minimal $\log$-resolution $\beta: V^{\prime} \rightarrow V$ of the pair $(V, B)$ is depicted in Fig. 18.

Proposition 6.3 For every pair $(\mu, v)$ such that $4 v-\mu= \pm 1$, the surface $S(\mu, \nu)=$ $V \backslash B$ is a $\mathbb{Z}$-acyclic fake plane of general type, whose real locus is birationally diffeomorphic to $\mathbb{R}^{2}$.

Proof By construction $\tau^{*} D=D+\mu A(p)+R$ and $\tau^{*} T_{p_{0}}(D)=T_{p_{0}}(D)+v A(p)+R^{\prime}$, where $R$ and $R^{\prime}$ are effective divisors supported on the union of $E_{1}, \ldots, E_{r-1}$. The free abelian group $\mathbb{Z}\langle B\rangle$ is generated by the classes of $T_{p_{0}}(D), E_{i}, \ldots, E_{r-1}$ and $D-4 T_{p_{0}}(D)$ while $\mathrm{Cl}(V)$ is freely generated by the classes of $T_{p_{0}}(D), E_{1}, \ldots, E_{r-1}$ and $A(p)$. Since $\tau^{*} D \sim 4 \tau^{*} T_{p_{0}}(D)$ in $\mathrm{Cl}(V)$, the matrix of the homomorphism $j_{*}: \mathbb{Z}\langle B\rangle \rightarrow \mathrm{Cl}(V)$ with respect to these bases has the form 


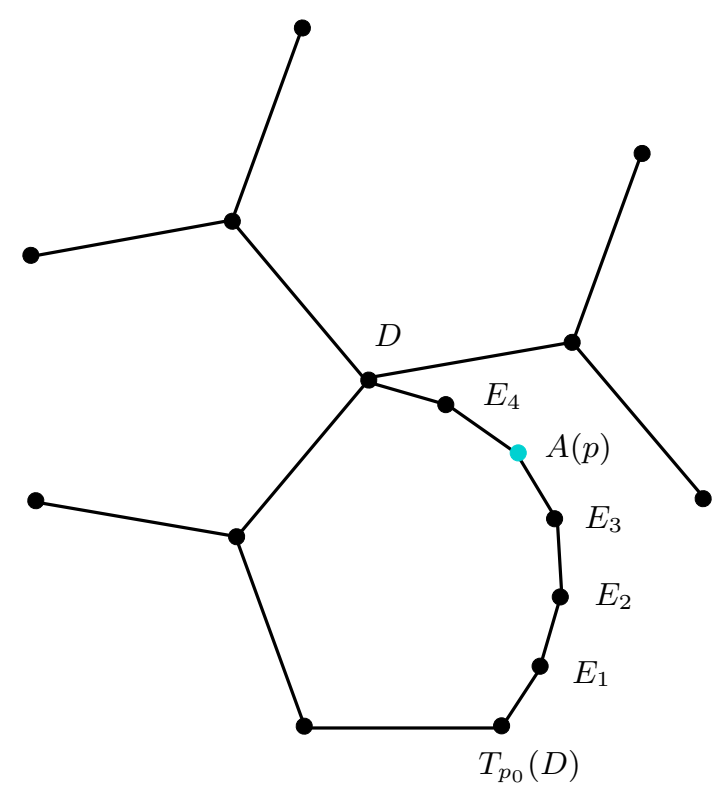

Fig. 18 Dual graph of the total transform of $B \cup A(p)$ in a minimal real $\log$-resolution $\beta: V^{\prime} \rightarrow V$ of the pair $(V, B)$ in the case $(\mu, v)=(7,2)$

$$
M=\left(\begin{array}{cc}
\operatorname{id}_{r+1} & * \\
0 & 4 v-\mu
\end{array}\right) \in \mathcal{M}_{r+2}(\mathbb{Z}) .
$$

The hypothesis that $4 v-\mu= \pm 1$ implies that $M \in \mathrm{GL}_{r+2}(\mathbb{Z})$ so that $S$ is $\mathbb{Z}$-acyclic by Theorem 2.1 (a). Furthermore, $H^{2}(G, \mathbb{Z}\langle B\rangle) \simeq \mathbb{Z}\langle B\rangle \otimes_{\mathbb{Z}} \mathbb{Z}_{2}, H^{2}(G, \mathrm{Cl}(V)) \simeq$ $\mathrm{Cl}(V) \otimes_{\mathbb{Z}} \mathbb{Z}_{2}$ as $\tau$ consists of the blow-ups of real points only, and the homomorphism $H^{2}\left(j_{*}\right)$ is represented by the reduction modulo 2 of $M$. Since $\mu$ is necessarily odd, $H^{2}\left(j_{*}\right)$ is thus an isomorphism and since $B(\mathbb{R})$ is not empty, we conclude from Theorem 2.1 (b) that $S$ is a real plane. The fact that $\kappa(S)=2$ follows from the same argument as in the proof of Proposition 6.1 using the structure of the weighted dual graph of the total transform of $B$ in a real minimal log-resolution $\beta: V^{\prime} \rightarrow V$ of the pair $(V, B)$ (see Fig. 18).

It remains to show that $S$ is $\mathbb{R}$-biregularly birationally equivalent to $\mathbb{C}^{2}$. Let $\theta: \mathbb{C P}^{2} \rightarrow \mathbb{C P}^{2}$ be the real birational involution consisting of the blow-up of the points $p_{0}$ with exceptional divisor $L \simeq \mathbb{P}_{\mathbb{R}}^{1}, q$ and $\bar{q}$ followed by the contraction of the proper transforms of the real line $L_{1} \simeq \mathbb{P}_{\mathbb{R}}^{1}$ passing through $q$ and $\bar{q}$ and of the pair of nonreal conjugate lines passing through $p_{0}$ and $q$ and $p_{0}$ and $\bar{q}$ respectively. The images of $\underset{\widetilde{T}}{D}, T_{p_{0}}(D)$ and $L$ by $\theta$ are respectively a smooth conic $\widetilde{D}$, a real line $\widetilde{T}$ intersecting $\widetilde{D}$ at $p$ and another real point $p_{\infty}$, and the tangent line $\widetilde{L}$ of $\widetilde{D}$ at $p_{\infty}$, see Fig. 19.

This map $\theta$ lifts to a real birational map $\widetilde{\theta}: V \rightarrow \widetilde{V}$ to the real projective surface $\widetilde{\tau}: \widetilde{V} \rightarrow \mathbb{C P}^{2}$ obtained from $\mathbb{C P}^{2}$ by blowing-up the real point $p_{1} \in \widetilde{L} \backslash(\widetilde{D} \cap \widetilde{L})$ on 

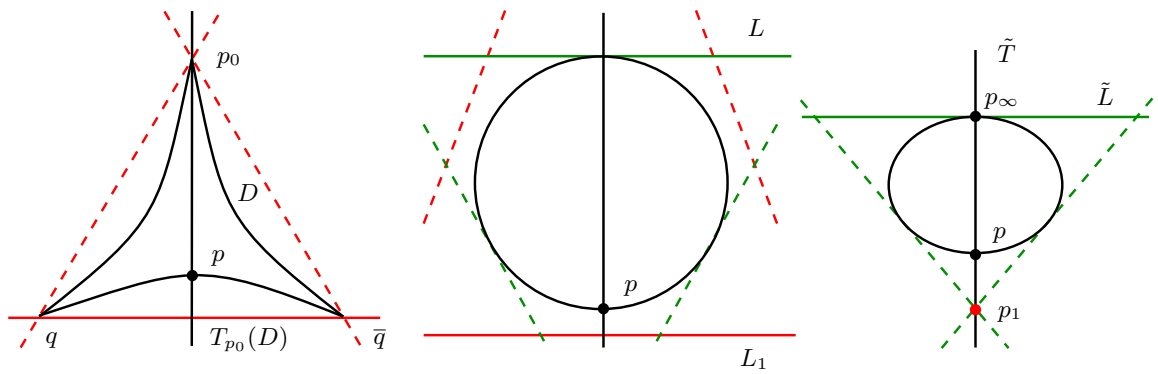

Fig. 19 Behavior of the tricuspidal quartic under the standard Cremona transformation
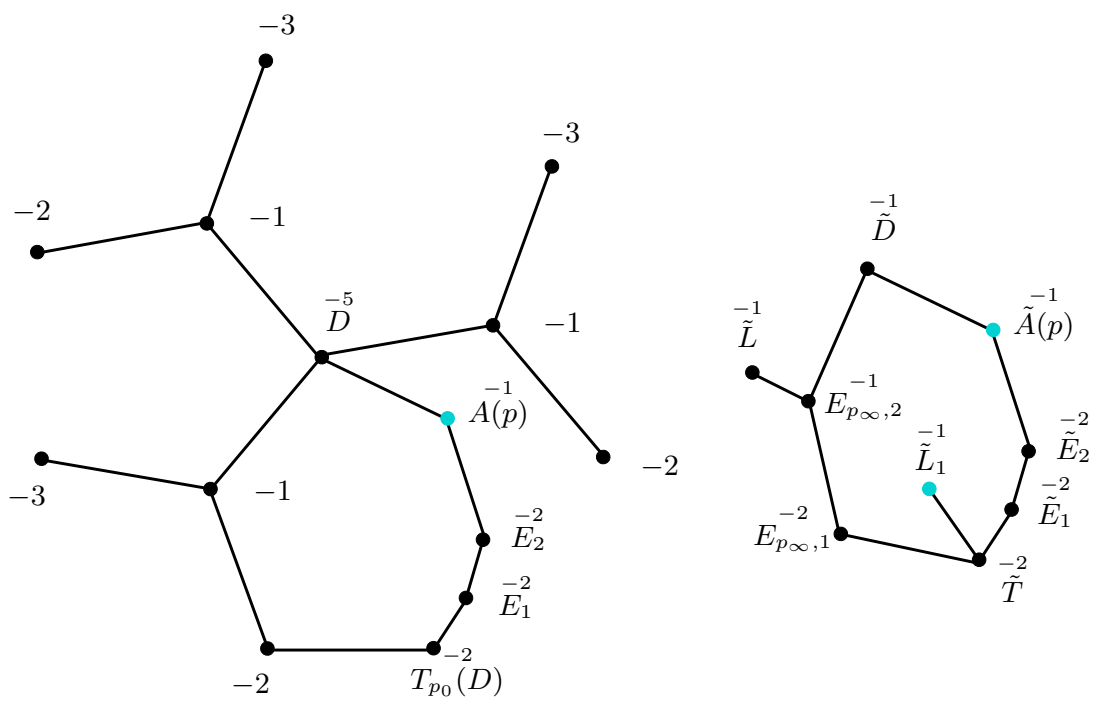

Fig. 20 Dual graphs of the total transforms of $B \cup A(p)$ and $\widetilde{B} \cup \widetilde{A}(p) \cup \widetilde{L}_{1}$ in a minimal $\log$-resolution of the pairs $(V, B)$ and $(\widetilde{V}, \widetilde{B})$ respectively for $(\mu, v)=(3,1)$

which $L_{1}$ was contracted and performing the same sequence of blow-ups as for the construction of $V$ over the point $p \in \widetilde{D} \cap \widetilde{T}$. Let $\widetilde{L}_{1}$ and $\widetilde{E}_{1}, \ldots, \widetilde{E}_{r-1}, \widetilde{E}_{r}=\widetilde{A}(p)$ be the corresponding exceptional divisors of $\widetilde{\tau}$ over $p_{1}$ and $p$ respectively and let $\widetilde{B}=\widetilde{D} \cup \widetilde{T} \cup \widetilde{L} \cup \cup_{i=1}^{r-1} \widetilde{E}_{i}$. Then $\widetilde{\theta}$ restricts to a diffeomorphism between the real loci of the surfaces $S$ and $\widetilde{S}=\widetilde{V} \backslash \widetilde{B}$. Using the relations

$$
\left\{\begin{array}{l}
\widetilde{\tau}^{*} \widetilde{T}=\widetilde{\tau}_{*}^{-1} \widetilde{T}+\widetilde{L}_{1}+v \widetilde{A}(p)+R_{1} \sim \widetilde{\tau}^{*} \widetilde{L} \\
\widetilde{\tau}^{*} \widetilde{D}=\widetilde{\tau}_{*}^{-1} \widetilde{D}+\mu \widetilde{A}(p)+R_{2} \sim 2 \widetilde{\tau}^{*} \widetilde{L}
\end{array}\right.
$$

in the divisor class group of $\widetilde{V}$, where $R_{1}$ and $R_{2}$ are effective divisors supported on $\bigcup_{i=1}^{r-1} \widetilde{E}_{i}$, we conclude that the homomorphism $\widetilde{j}_{*}: \mathbb{Z}\langle\widetilde{B}\rangle \rightarrow \mathrm{Cl}(\widetilde{V})$ induced by the inclusion $\widetilde{B} \hookrightarrow \widetilde{V}$ is represented in appropriate bases by a matrix of the form 


$$
\widetilde{M}=\left(\begin{array}{ccc}
\operatorname{id}_{r+1} & * & * \\
0 & 0 & -1 \\
0 & -\mu & -v
\end{array}\right)
$$

Since $4 v-\mu= \pm 1, \mu$ is odd, $\widetilde{M} \in \mathrm{GL}_{r+3}(\mathbb{Q})$, and we deduce from Theorem 2.1 (a) and Remark 2.2 that $\widetilde{S}$ is $\mathbb{Q}$-acyclic, with $H_{1}(\widetilde{S}, \mathbb{Z}) \simeq \mathbb{Z} \mu$. So $\widetilde{S}$ is in particular affine. Furthermore, the proper transform of $\widetilde{L}$ in a minimal real $\log$-resolution $\widetilde{\beta}: \widetilde{V}^{\prime} \rightarrow \widetilde{V}$ of the pair $(\widetilde{V}, \widetilde{B})$ is a $(-1)$-curve. Contracting it, we obtain a completion of $\widetilde{S}$ into a smooth real projective surface $\left(\widetilde{V}^{\prime \prime}, \widetilde{B}^{\prime \prime}\right)$ whose boundary $\widetilde{B}^{\prime \prime}$ is a chain of rational curves. It follows from [10] that $\widetilde{S}$ admits an $\mathbb{A}^{1}$-fibration over $\mathbb{C}$. So $\kappa(\widetilde{S})=-\infty$, and we then deduce from Theorem 3.1 that $\widetilde{S}$ admits a real $\mathbb{A}^{1}$-fibration $\widetilde{\rho}: \widetilde{S} \rightarrow \mathbb{C}$. By [7, Proposition 2.15], $\widetilde{\rho}$ has at most one degenerate fiber, and so, $\widetilde{S}$ is $\mathbb{R}$-biregularly equivalent to $\mathbb{C}^{2}$ by virtue of Theorem 3.3 . This completes the proof.

Example 6.4 In the simplest case $(\mu, v)=(3,1)$, the dual graph of the total transform of $\widetilde{L}_{1} \cup \widetilde{B} \cup \widetilde{A}(p)$ in the minimal $\log$-resolution $\widetilde{\beta}: \widetilde{V}^{\prime} \rightarrow \widetilde{V}$ of the pair $(\widetilde{V}, \widetilde{B}=$ $\left.\widetilde{D} \cup \widetilde{T} \cup \widetilde{L} \cup \widetilde{E}_{1} \cup \widetilde{E}_{2}\right)$ is depicted on the right hand side of Fig. 20, where $E_{p_{\infty}, 1}$ and $E_{p_{\infty}, 2}$ denote the two exceptional divisors of $\widetilde{\beta}$ over $p_{\infty}$. Let $\gamma: \widetilde{V}^{\prime} \rightarrow \widetilde{V}^{\prime \prime}$ be the real birational map consisting of the blow-up of the real point $E_{p_{\infty, 1}} \cap E_{p_{\infty}, 2}$ with exceptional divisor $C$, followed by the contraction of $\widetilde{L}$ and $\widetilde{D}$. The total image $\widetilde{B}^{\prime \prime}=E_{p_{\infty}, 2} \cup C \cup E_{p_{\infty}, 1} \cup \widetilde{T} \cup \widetilde{E}_{1} \cup \widetilde{E}_{2}$ of $\widetilde{B}^{\prime}$ by $\gamma$ is a chain of rational curves with self-intersections $(0,-1,-3,-2,-2,-2)$. The complete linear system $\left|E_{p_{\infty, 2}}\right|$ on $\widetilde{V}^{\prime \prime}$ defines a real $\mathbb{P}^{1}$-fibration $\bar{\rho}: \widetilde{V}^{\prime \prime} \rightarrow \mathbb{C P}^{1}$ having $C$ has a section and $E_{p_{\infty, 1}}+$ $3 \widetilde{T}+3 \widetilde{L}_{1}+2 \widetilde{E}_{1}+\widetilde{E}_{2}$ as a unique degenerate fiber. The restriction of $\bar{\rho}$ to $\widetilde{S} \simeq \widetilde{V}^{\prime \prime} \backslash \widetilde{B}^{\prime \prime}$ is a real $\mathbb{A}^{1}$-fibration $\widetilde{\rho}: \widetilde{S} \rightarrow \mathbb{C}$ having $\widetilde{L}_{1} \cap \widetilde{S} \simeq \mathbb{C}$ has unique degenerate fiber, of multiplicity 3 .

\section{References}

1. Bertin, J.: Pinceaux de droites et automorphismes des surfaces affines. J. Reine Angew. Math. 341, 32-53 (1983)

2. Blanc, J., Dubouloz, A.: Automorphisms of $\mathbb{A}^{1}$-fibered surfaces. Trans. Amer. Math. Soc. 363(11), 5887-5924 (2011)

3. Borel, A., Haefliger, A.: La classe d'homologie fondamentale d'un espace analytique. Bull. Soc. Math. France 89, 461-513 (1961)

4. tom Dieck, T., Petrie, T.: Contractible affine surfaces of Kodaira dimension one. Japan J. Math. 16(1), 147-169 (1990)

5. tom Dieck, T., Petrie, T.: Homology planes and algebraic curves. Osaka J. Math. 30(4), 855-886 (1993)

6. Dimca, A.: Singularities and Topology of Hypersurfaces. Universitext. Springer, New York (1992)

7. Dubouloz, A.: Completions of normal affine surfaces with a trivial Makar-Limanov invariant. Michigan Math. J. 52(2), 289-308 (2004)

8. Dubouloz, A., Mangolte, F.: Fake real planes: exotic affine algebraic models of $\mathbb{R}^{2}(2015)$. arXiv: 1507.01574

9. Fujita, T.: On the topology of noncomplete algebraic surfaces. J. Fac. Sci. Univ. Tokyo Sect. IA Math. 29(3), 503-566 (1982)

10. Gizatullin, M.H.: Quasihomogeneous affine surfaces. Math. USSR-Izv. 5(5), 1057-1081 (1971)

11. Gurjar, R.V., Miyanishi, M.: Affine surfaces with $\bar{\kappa} \leqslant 1$. In: Baily, W.L., Shioda, T. (eds.) Algebraic Geometry and Commutative Algebra, I, pp. 99-124. Kinokuniya, Tokyo (1987)

12. Gurjar, R.V., Pradeep, C.R.: $\mathbb{Q}$-homology planes are rational III. Osaka J. Math. 36(2), 259-335 (1999) 
13. Gurjar, R.V., Pradeep, C.R., Shastri, A.R.: On rationality of logarithmic $\mathbb{Q}$-homology planes-II. Osaka J. Math. 34(3), 725-743 (1997)

14. Kambayashi, T.: On the absence of nontrivial separable forms of the affine plane. J. Algebra 35, 449-456 (1975)

15. Kulikov, Vik.S., Kharlamov, V.M.: On real structures on rigid surfaces. Izv. Math. 66(1), 133-152 (2002)

16. Mangolte, F.: Real rational surfaces. In: Real Algebraic Geometry. Panoramas et Synthèses (2016, to appear)

17. Miyanishi, M.: Open Algebraic Surfaces. CRM Monograph Series, vol. 12. American Mathematical Society, Providence (2001)

18. Miyanishi, M., Sugie, T.: Affine surfaces containing cylinderlike open sets. J. Math. Kyoto Univ. 20(1), $11-42$ (1980)

19. Namba, M.: Geometry of Projective Algebraic Curves. Monographs and Textbooks in Pure and Applied Mathematics, vol. 88. Marcel Dekker, New York (1984)

20. Ramanujam, C.P.: A topological characterisation of the affine plane as an algebraic variety. Ann. Math. 94(1), 69-88 (1971)

21. Wakabayashi, I.: On the logarithmic Kodaira dimension of the complement of a curve in $\mathbb{P}^{2}$. Proc. Japan Acad. Ser. A Math. Sci. 54(6), 157-162 (1978)

22. Zaidenberg, M.: Exotic algebraic structures on affine spaces. St. Petersburg Math. J. 11(5), 703-760 (2000) 